Portland State University

PDXScholar

$1-19-1977$

\title{
Unwiederbringlich und Effi Briest : Eine Studie zu Theodor Fontanes Erzählweise
}

Christine Gentzkow

Portland State University

Follow this and additional works at: https://pdxscholar.library.pdx.edu/open_access_etds

Part of the German Language and Literature Commons Let us know how access to this document benefits you.

Recommended Citation

Gentzkow, Christine, "Unwiederbringlich und Effı Briest : Eine Studie zu Theodor Fontanes Erzählweise" (1977). Dissertations and Theses. Paper 2560.

https://doi.org/10.15760/etd.2557

This Thesis is brought to you for free and open access. It has been accepted for inclusion in Dissertations and Theses by an authorized administrator of PDXScholar. Please contact us if we can make this document more accessible: pdxscholar@pdx.edu. 
AN ABSTRACT OF THE THESIS OF Christine Gentzkow for the Master of Arts in German presented January 19, 1977.

Title: Unwiederbringlich und Effi Briest:

Eine Studie zu Theodor Fontanes Erzählweise.

APPROVED BY MEMBERS OF THE THESIS COMMTTTEE:

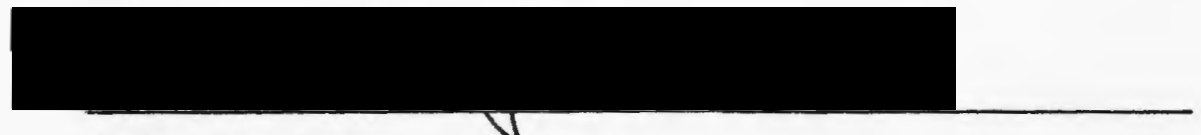

Fránz Langhammer, Chairman

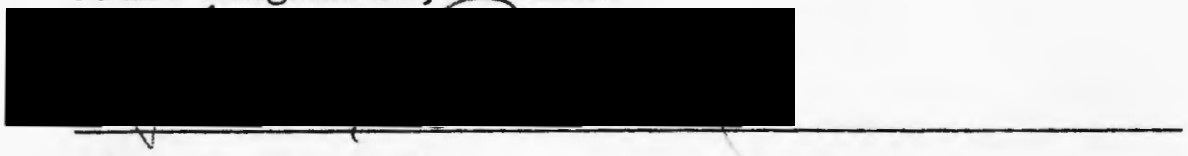

Linda B. Parshall

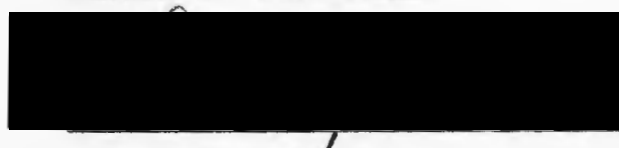

Louis J. Elteto

In this thesis an attempt is made to show the peculiar features of Fontane's narrative style as well as his art of characterization. I have tried to interpret his concept of humaneness in the two novels, Unwiederbringlich and Effi Briest, and to show how the composition of these novels is directed to the portrait of the individual.

Theodor Fontane's unusual distinction lies in his keen observation of the Prussian scene and his craftsmanship as a cosmopolitan storyteller. The principal elements of his works are a sense of historical continuity and an uncommon perception of the speech and gestures by which his characters reveal their particular virtues. In his early writings about his travels abroad and his excursions through the 
province of Brandenburg, he developed powers of observation and a narrative style which served him well in his major novels. His composition is picturesque. The plots are enlivened with anecdotes and surprising detail; the portraiture is subtle and precise. Fontane's portrayal of human relationships takes into account the impact of coincidences of life. His symbolism, apparent not only to his readers, but also to his characters, is simple but effective.

Fontane seems to favor particularly the individual who is restricted by conventions and whose conflicts derive from these restrictions. His characters, like himself, are caught between the meaningless traditions of former times and the dawn of a new awareness. 
UNWIEDERBRINGLICH UND EFFI BRIEST:

EINE STUDIE ZU THEODOR FONTANES ERZÄHLWEISE

by

CHRISTINE GENTZKOW

A thesis submitted in partial fulfillment

of the requirements for the degree of

MASTER OF ARTS

in

GERMAN

Portland State University

1977 
TO THE OFFICE OF GRADUATE STUDIES:

The members of the Committee approve the thesis of Christine Gentzkow presented January 19, 1977.

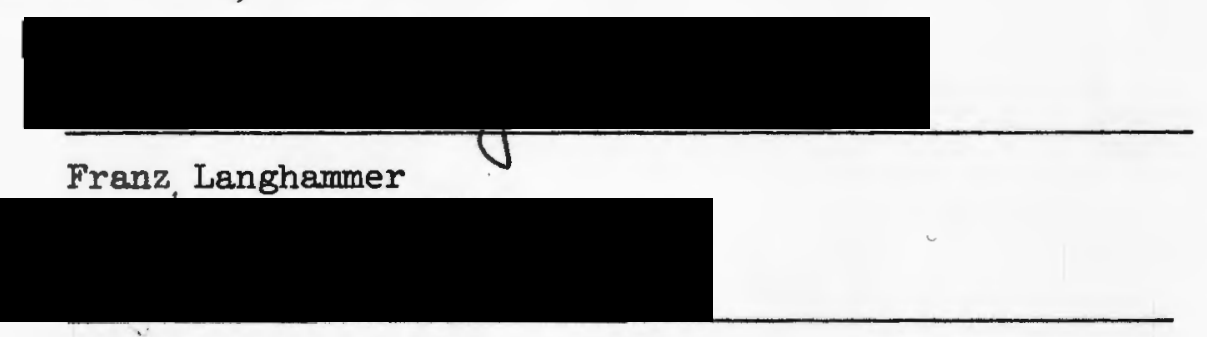

Iinda B. Parshall

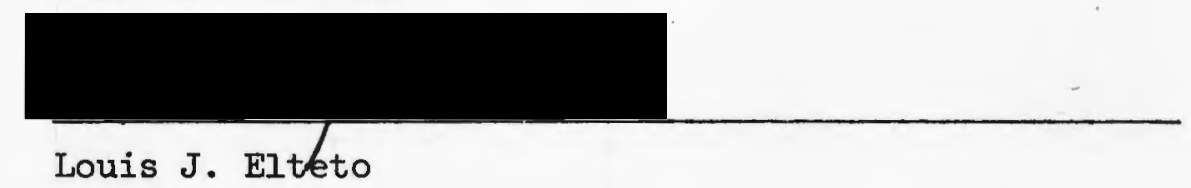

APPROVED :

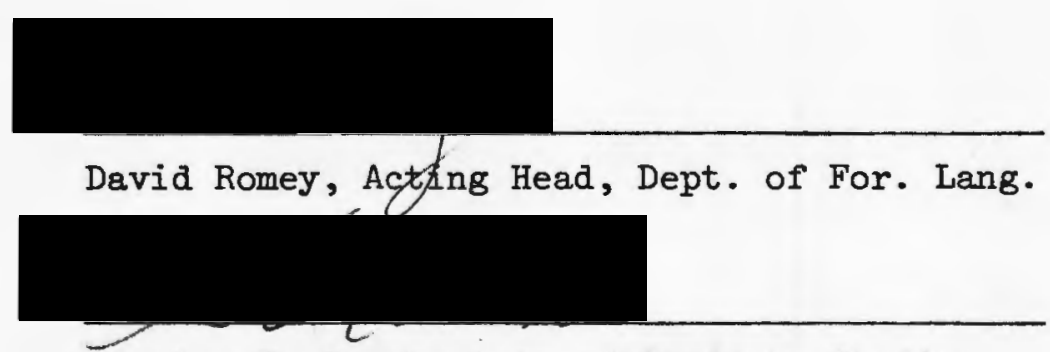

Stanley E. Rauch, Dean of Graduate Studies

February 25, 1977 
INHAIT

\section{EINLEITUNG}

ÜBERBLICK . . . . . . . . . . . . . . . . . . . . . 1 KAPITEL I

DER WIEDERENTDECKTE FONTANE . . . . . . . . . . . . . 6 BESPRECHUNG VON UNWIEDERBRINGLICH UNTER DEM GESICHTSPUNKT DER VORDEUTUNG . . . . . . . . . . . . . . . T HOLK UND CHRISTINE: STUDIE ZUR VERSCHIEDENHETT DER CHARAKTERE . . . . . . . . . . . 19 KAPITEL II

VORGESCHICHIE ZU EFFI BRIEST . . . . . . . . . . . . . 25 BESPRECHUNG VON EFFI BRIEST UNTER DEM GESICHTSPUNKT DER VORDEUTUNG . . . . . . . . . . . . . . 27 EFFI UND ININSTETTEN: STUDIE ZUR VERSCHIEDENHEIT DER CHARAKTERE . . . . . . . . . . . . 33 VERGLEICH DER HAUPTFIGUREN: CHRISTINE - INNSTETTEN, EFFI - HOLK . . . . . . . . . . . 39

KAPITEL III

THEODOR FONTANES ROMANTHEORTE . . . . . . . . . . . . 42 DIE KUNSTMITTEL IN DEN ROMANEN UNWIEDERBRINGLICH

UND EHFI BRIEST: GESPRÄCH, SPRACHE, WORT, BRIEF . . . . . . 44 ÖRTLICHKEIT, SYMBOL, LEITMOTIV . . . . . . . . . . . . . 54 SCHLUB

RESIGNATION ALS AUSDRUCKSFORM DER MENSCHLICHKEIT . . . . . . 60 FUBNOTEN . . . . . . . . . . . . . . . . . . 63 LITERATURVERZEICHNIS . . . . . . . . . . . . . . . 65 
EINLEITUNG

ÜBERBLICK

Das erste Drittel des 19. Jahrhunderts hat viele bedeutende deutsche Erzähler hervorgebracht. Zu ihnen gehört Theodor Fontane.

Am Anfang dieser Erzählergeneration steht Adalbert Stifter (18051868). Ihm folgen gut ein Jahrzehnt später Gustav Freytag (1816-1895), Theodor Storm (1817-1888), Gottfried Keller (1819-1890) und Theodor Fontane (1819-1898). Schließlich kommen im dritten Jahrzehnt Konrad Ferdinand Meyer (1825-1898) und Wilhelm Raabe (1831-1910) dazu.

Theodor Fontane nimmt also chronologisch eine Mittelstellung ein. Und doch unterscheidet er sich von allen Genannten, wenn wir nicht die Lebensdauer, sondern die dichterische Schaffensperiode ansehen.

Adalbert Stifters Werk war beendet. Gustav Freytag arbeitete an seinem letzten Roman Die Ahnen. Kellers Der grüne Heinrich, Die Leute von Seldwyla und Züricher Novellen waren erschienen. Theodor Storms Immensee, Viola tricolor und Aquis submersus waren veröffentlicht. Selbst der jüngere Wilhelm Raabe hatte schon Die Chronik der Sperlingsgasse, Der Hungerpastor und Abu Telfan geschrieben, und Conrad Ferdinand Meyer hatte sich mit seinem Versepos Huttens letzte Tage einen Namen gemacht.

Theodor Fontane jedoch hatte dem Schaffen seiner Zeitgenossen nichts Ebenbürtiges zur Seite zu stellen. Er war nur als Dichter von Balladen, als Journalist, Essayist und Autor der Wanderungen durch die Mark Brandenburg bekannt. Seine Generationsgefährten standen auf dem 
Höhepunkt ihres Schaffens oder hatten diesen schon überschritten, als Fontanes erster Roman Vor dem Sturm 1878 erschien. In den verbleibenden zwanzig Jahren seines Lebens schrieb er dann nicht weniger als sechzehn Novellen und Romane, übertraf das Werk der Altersgenossen und etablierte sich als der Schriftsteller des Gesellschaftsromans. Die Sonderstellung, die Fontane in der Erzählkunst des 19: Jahrhunderts einnimmt, liegt also zunächst in seiner Verspätung, dann in der Schaffensfülle und schließlich in seiner. Verfrühung.

Die rein zeitliche Verspätung überrascht nur, wenn man Fontane wieder mit seinen Altersgenossen vergleicht. Doch gemessen an Fontanes Gesamtwerk erhält der Begriff Verspätung eine ganz bestimmte Bedeutung. Verspätung soll heib́en: nicht die Balladen, nicht die historischen und landschaftlichen Beschreibungen, nicht die kritischen Arbeiten waren seine eigentliche Berufung, sondern die Erzählung, die dichterische Darstellung des Menschen gegen den Hintergrund des Historischen und Landschaftlichen. Denn in dieser Darstellung liegt Fontanes Meisterschaft. Alles was an schriftstellerischer Tätigkeit vorausgegangen war, muß als Vorbereitung angesehen werden. Überraschend ist also eher die lange Vorbereitungszeit und nicht so sehr die zeitliche Verspätung. Welchen Gewinn Fontane für seine Romane aus dieser langen Vorbereitung zog, ist offensichtlich. Die genaue Kenntnis der Landschaft, die er sich in den Wanderungen angeeignet hatte, findet in den Berliner Romanen einen tiefen Niederschlag; und die verschiedenen schriftstellerischen Mittel: Kritik, Bericht, Erzählung, Brief, Gespräch, die er in seinen Romanen anwendet, waren über mehrere Jahrzehnte erprobt und verfeinert worden. 
Verspëtung will aber auch Distanz heißen, denn vor allem das Alter kann dem Leben mit Distanz begegnen ohne teilnahmslos am menschlichen Schicksal zu werden.

Ausgestattet mit gründlicher Kenntnis der Landschaft, mit Sinn für das Geschichtliche, mit erprobtem kritischem Vermögen, mit scharfer Beobachtungsgabe für den Mitmenschen und mit der nötigen Distanz, schafft der Verspätete seine dichterischen Werke.

Die Sonderstellung, die Fontane in der literarischen Produktivität des 19. Jahrhunderts einnimmt, liegt nicht so sehr in der Anzahl der Romane und Novellen sondern vielmehr im Inhalt und Gehalt seines Werkes.

Sein erster Roman Vor dem Sturm (1878) behandelt das Leben im Schatten der großen historischen Ereignisse von 1812-13. Fontane zeigt nicht die weltgeschichtlichen Veränderungen und Katastrophen, sondern die fast trivialen Begebenheiten, die das Leben des Menschen bestimmen. In den kurzen Novellen Grete Minde (1880), Ellernklipp (1881) und Schach von Wuthenow (1883) befaßst sich Fontane seiner eigenen Aussage nach mit "Charakteren" und mit dem Verhältnis von "Schuld und Strafe." Unterm Birnbaum (1885) ist eine Kriminalnovelle, deren Spannung dadurch erzeugt wird, daß der Leser zwischen Mitgefühl für den Täter und Abscheu für seine Handlung schwankt. Fontanes zweite Kriminalnovelle Quitt (1885 geschrieben, 1891 veröffentlicht) ist ihm nicht so gut gelungen. Das mag zum Teil daran liegen, daß Fontane nicht mit der Örtlichkeit der Handlung vertraut war (Quitt spielt in Schlesien und teilweise in Amerika), und deshalb mußte er zu anderen Mitteln der Charakterdarstellung greifen. Zum Teil aber liegt es wohl auch daran, daß man spürte, wie sehr Fontane auf sittlicher Ordnung besteht. 
Der Roman Graf Petöfy (1884) zählt aus dem gleichen Grunde wie Quitt nicht zu den besseren Arbeiten Fontanes. Petöfy spielt in Wien und Ungarn.

In den folgenden vier Romanen behandelt Fontane die the und ihre Probleme. I'Aldultera (1882) kann als Vorläufer betrachtet werden und ist nicht, wie die drei anderen, eine Tragödie. Cecile (1887), Unwiederbringlich (1892) und Effi Briest (1895) dagegen enden tragisch. In diesen drei Romanen führt der Ehebruch mit seinen Folgen für das Leben der Heldin zur Katastrophe.

Zu den Tragödien Fontanes kann man auch die Romane Stine (1890) und Irrungen, Wirrungen (1888) zählen. Hier handelt es sich um die Liebe zweier.Menschen, die verschiedenen Ständen engehören, und aus diesem Grunde führt ihre Liebe nicht zu einer legitimen Verbindung, sondern endet in Entsagung.

Fontane hat aber auch Komödien geschrieben und sich ebenfalls hier als Meister erwiesen. Frau Jenny Treibel (1892) stellt den Beweis dafür. Die beiden anderen Romane sind Mathilde Möhring (1891 geschrieben, 1908 veröffentlicht) und Die Poggenpuhls (1896). Mit Humor und Ironie zeichnet Fontane seine Gestalten und deckt ihre Schwächen auf.

Fontanes letzter Roman erinnert in Länge und Breite an seinen ersten. Der Stechlin (1898) hat wenig Handlung und stellt im Wesentlichen den Ausklang einer alten und die Vorzeichen einer neuen Gesellschaft dar.

Es hat mehrere deutsche Erzähler im 19. Jahrhundert gegeben, die zwei oder drei außerordentliche Werke hervorgebracht haben, aber keinem ist es wie Fontane gelungen, ein Bild der Gesellschaft entstehen zu lassen. Es war nicht Fontanes Absicht, die Gesellschaft zu verändern. 


\section{KAPITEL I}

\section{DER WIEDERENTDECKT'E FONTANE}

"Gott, wer liest Novellen bei der Hitze? Wer hat jetzt Lust und Fähigkeit auf die hundert und, ich kann dreist sagen, auf die tausend Finessen zu achten, die ich dieser von mir besonders geliebten Arbeit mit auf den Lebensweg gegeben habe?" 3 . So fragte Fontane im Sommer 1887 in einem Brief, als er an seinem Gesellschaftsroman Irrungen, Wirrungen arbeitete.

Heute, 90 Jahre später, scheint diese skeptische Frage mehr Berechtigung zu haben als damals. Eine eindeutige Antwort läßt sich nicht leicht finden. Doch übersieht man die Darstellungen zur FontaneLiteratur, überrascht die Vielzahl der Arbeiten. ${ }^{4}$ Besonders in der zweiten Hälfte des 20. Jahrhunderts häufen sich die kritischen Untersuchungen, die dem dichterischen Schaffen Fontanes gerecht zu werden versuchen.

Überraschend ist, diese Tatsache vor allem deshalb, weil sich die gesellschaftliche Struktur des 19. Jahrhunderts, die in den Werken Fontanes die Basis der menschlichen Existenz ist, durch die historischen Ereignisse dieses Jahrhunderts grundlegend verändert hat. Der Adel, die bevorzugte Gesellschaftsschicht in den Erzählungen, existiert heute nicht mehr. Veraltet scheint auch der Konflikt, der sich aus der Zugehörigkeit verschiedener Gesellschaftsklassen entwickelte. Die Gesellschaftskritik, die Fontane in seinen Romanen übt, kann nur noch historisches Interesse erwecken. 
Er wollte ihr nur den Spiegel vorhalten.

Umgebung, Umstände und Zeit bestimmen das Handeln seiner Gestalten, aber ihre Freuden, Hoffnungen und Schmerzen erwecken in uns heute noch das gleiche Mitgefühl und Verständnis, das der Dichter ihnen entgegenbrachte.

Welche Bedeutung das Werk Fontanes in der Geschichte der deutschen Literatur spielen sollte, ist erst nach und nach im 20. Jahrhundert deutlich geworden. Er hat nicht die Traditionen seiner Generation fortgesetzt, sondern sie überwunden, und den Erzählern der folgenden Generation den Weg gewiesen. Darin liegt seine Verfrühung.

Seine Zeitgenossen beurteilten sein Werk ihren eigenen Weltanschauungen gemäB - entweder mit Bewunderung oder Ablehnung. Für die einen ist Fontane ein "Berliner Lokalpatriot," dessen "Sittenbilder" sich auf das Publikum seiner engeren Heimat beschränken. Andere sehen seine Bedeutung vielmehr in seiner Stellung zum Naturalismus. Wieder andere meinen, er hätte "die Wege der Unsittenschilderung eingeschlagen." Allgemein anerkannt wird aber die "Wirklichkeit seiner Schilderung."

Die neuere Fontaneforschung hat jedoch das Werk des Dichters objektiver beurteilt. Stellvertretend soll hier Hans-Heinrich Reuter zu Wort kommen wenn er darauf hinweist, was Fontanes Alterswerk auszeichnete:

Zukunftsträchtigkeit, Echtheit, Wahrheit, Lebensfülle... Beobachtung des Menschen, Sinn für die Geschichte, Verantwortung und Mut zur Kritik: der reife Realismus des gesellschaftlichen Schriftstellers Fontane gründet sich in dieser ein Leben lang bewahrten, erprobten und vervollkommeten, sich wechselseitig bedingenden Dreiheit, beruht auf ihrer Erweiterung vom Menschen auf die Gesellschaft, von der Geschichte auf die Gegenwart und die Zukunft, von der Kritik auf die Forschung, zuletzt auf die Vision.? 
Um so größer ist aber das Interesse der Literaturforschung an den "tausend Finessen." Was Paul Schlenther 1892 in seiner Rezension über Unwiederbringlich geschrieben hat, ist wiederentdeckt worden:

Ist man einmal dieser verschnitzten Dichtermethode auf die Spur gekommen, so gewährt es das hellste ästhetische Vergnügen, einem sonderbar schaffenden, reichen und tiefen Geist auf all seinen Schleichwegen und kleinen feinen Winkelzügen zu folgen. Fontanes Dichtungen geben sich daher nicht aus, wenn die erste Spannung befriedigt ist. Man muß sie um- und umwenden, und oft wird erst der Anfang durchs Ende ganz begreiflich. 5

Diese Arbeit will versuchen, an Hand von zwei Romanen der neunziger Jahre, Unwiederbringlich und Effi Briest, die Besonderheiten der Fontaneschen Erzählweise und ihre Charakteresierungskunst aufzuzeigen. Bestimmend für diese Untersuchung ist die Frage, was der Erzähler Fontane als das Humane sieht, denn er sagte selbst: "Der Mensch bleibt in aller Kunst doch immer die Hauptsache." 6

BESPRECHUNG VON UNWIEDERBRINGLICH UNTER DEM GESICHTSPUNKT DER VORDEUTUNG

In seinem Roman Unwiederbringlich zeigt Theodor Fontane den Zerfall einer the.

Graf Holk und Baronesse Christine Arne hatten vor siebzehn Jahren geheiratet und führten eine glückliche Ehe. Die kleinen Differenzen, die in Erziehungs- und religiösen Fragen zwischen ihnen bestanden, gefährdeten ihr Glück nicht: Doch seit die Kinder herangewachsen waren, häuften sich die Meinungsverschiedenheiten.

Hier setzt die eigentliche Handlung des Romans ein. Graf Holk wird an den Hof in Kopenhagen berufen. Die Trennung ist den Eheleuten willkommen. In Kopenhagen lernt Holk die Hofdame Ebba von Rosenberg kennen und verliebt sich in sie. Um Ebba heiraten zu können, läßt er 
sich von Christine scheiden. Christine geht nach Gnadenfrei, wo sie ihre Mädchenjahre im Pensionat verbracht hatte. Ebba weist Holks Heiratsantrag zurück. Graf Holk verlä:st Kopenhagen. Mehrere Monate reist er ziellos durch Europa und läßt sich schlieBlich in London nieder. Christines Bruder Arne und Pastor Schwarzkoppen, ein Freund der Familie, vermitteln zwischen den geschiedenen Eheleuten. Es kommt zu einer Wiedervereinigung. Doch die the dauert nur einige Monate und endet mit Christines Selbstmord.

Die Handlung des Romans beruht auf einer tatsächlichen Begebenheit. In einem Brief vom 21. November, 1888 an Julius Rodenberg berichtet Fontane folgende Geschichte, die ihm eine Bekannte vor drei, vier Jahren mitgeteilt hatte:

Baron Plessen-Ivenack auf SchlOB Ivenack in Strelitz, Kavalier comme il faut, Ehrenmann, lebte seit 18 Jahren in einer glücklichen Ehe. Die Frau 37, noch schön, etwas fromm (die Strelitzer tun es nicht anders). Er Kammerherr. Als solcher wird er zu vorübergehender Dienstleistung an den Strelitzer Hof berufen. Hier macht er die Bekanntschaft eines jungen pommerschen Fräuleins v. Dewitz, eines Ausbundes nicht von Schönheit, aber von Pikanterie. Den Rest brauche ich Ihnen nicint zu erzählen. Er ist behext, kehrt nach Ivenack zurück und sagt seiner Frau: sie müßten sich trennen, so und so. Die Frau, tödlich getroffen, willigt in alles und geht. Die Scheidung wird gerichtlich ausgesprochen. Und nun kehrt der Baron nach Strelitz zurück und wirbt in aller Form um die Dewitz. Die lacht ihn aus. Sie steht eben auf dem Punkte, sich mit einem ebenso reichen, aber unverheirateten Herrn aus der Strelitzer Gesellschaft zu verloben. Der arme Karl, er hat die Taube auf dem Dach gewollt und hat nun weder Taube noch Sperling. Alles weg. Er geht ins Ausland, ist ein unglücklicher, blamierter und halb dem Ridikül verfallener Mann. Inzwischen aber ist die älteste Tochter, die beide Eltern gleich schwärmerisch liebt, herangewachsen, es spielen allerhand Szenen in der Verwandtschaft, Versöhnungsversuche drängen sich, und das Ende vom Liede ist: es soll alles vergessen sein. Zwei Jahre sind vergangen. Die Frau willigt ein, und unter nie dagewesener Pracht, darin sich der Jubel des ganzen Landes Strelitz mischt, wird das geschiedene Paar zum zweiten Male getraut. Alles steht Kopf, der Hof nimmt teil, Telegramme von Gott weib woher, Musik und Toaste. 
Plötzlich aber ist die Wiedergetraute, die wieder Strahlende, die wieder scheinbar Glückliche von der Seite ihres Mannes verschwunden, und als man nach ihr sucht, findet man sie tot am Teich. Und auf ihrem Zimmer einen Brief, der nichts enthält als das Wort: Unwiederbringlich.7

Aus Rücksicht auf die betroffene Familie verlegte Fontane den Schauplatz der Handlung nach Nordschleswig und Kopenhagen. Diese Transponierung des geographischen Ortes verlagerte den Schwerpunkt des Romans von der vorwiegend gesellschaftskritischen Sphäre in die menschlich-persönliche. Strelitz wäre ihm lieber gewesen und hätte seiner "Geschichte den Ton des politisch Satirischen gegeben: nun klingt nordisch Romantisches durch." (R.E., Bd. VI, S. 464). Der Roman behandelt deshalb nicht den Konflikt zwischen Individuum und Gesellschaft, sondern den ehelichen Konflikt diskrepanter Charaktere.

"Unwiederbringlich," das Schlußwort der oben angeführten Skizze, stellt Fontane als enthüllenden Titel seinem Roman voran. So hat er schon mit dem ersten Wort den Leser deutlich auf das Ende vorbereitet. Er will also die Entwicklung der Ereignisse, die im tragischen Selbstmord gipfeln, vom Leser mitgeahnt und mitverstanden wissen.

Verfolgen wir im einzelnen den Weg, den Fontane mit seinem Titel gewiesen hat.

Im ersten Kapitel berichtet der Erzähler von dem Schlo3, das die Holksche Familie bewohnt. Es wird mit einem "Tempel" verglichen. Damit ist der Hinweis auf Religion und Opfer gegeben. Die Religion bestimmt Christines Denken und Handeln. Der Bezug von "Tempel" zu "Opfer" weist deutlich auf ihren Tod voraus.

Im zweiten Teil des ersten Kapitels kontrastiert Fontane das alte Schloß mit dem Neubau. Christine wäre lieber an alter stelle verblieben. Holk aber verfolgt eigensinnig seine Baupläne für das "Schloß am Meer," 
wie er es nach einem Uhlandschen Liede benennt. Holk erhofft für beide ein größeres Glück in ihrer neuen Wohnung. Christine verweist ihn aber auf das traurige Ende des von ihm zitierten Gedichtes und meint: "Wenn man glücklich ist, soll man nicht noch glücklicher sein wollen." (R.E., Bd. 6, S. 9).

Diese kleine Szene deutet auf die grundliegenden Unterschiede zwischen den Ehegatten. Christine fühlt sich in der alten Ungebung geborgen und fürchtet sich vor den Veränderungen. Holk dagegen legt wenig Gewicht auf Christines Vorahnungen und freut sich der neuen Bequemlichkeiten.

Weitere sieben glückliche Jahre vergehen und scheinen Christines Befürchtungen zu wiederlegen. Die Harmonie des Hauses wird nur selten durch Auseinandersetzungen gestört. Doch in letzter Zeit häufen sich die Differenzen und im zweiten Kapitel wird der Konflikt in seiner ganzen Schwere dargestellt. Wir sehen das Enepaar Holk im Kreise ihrer Freunde und Verwandten. Holk und Arne, Christines Bruder, gehen auf und $a b$ und diskutieren die neuesten Erkenntnisse auf dem Gebiet der Viehpraxis. Christine dagegen sitzt mit ihrer Gesellschafterin Dobschütz und Seminardirektor Schwarzkoppen auf der Terrasse. Sie besprechen zunächst Schul- und Erziehungsfragen und schließlich Christines Lieblingsthema, die Pläne für die Errichtung einer Familiengruft. Als sich am Ende des Kapitels die beiden Gruppen zusammenfinden, wird aus dem Gespräch ein Wortwechsel. Christine zeigt kein Verständnis für Holks landwirtschaftliche Begeisterung. Holk antwortet ihr bitter und spöttisch, daỉ sie nur für die Kirche und Grabstätten Interesse zeige. Die Bitterkeit, mit der hier die Vorwürfe auf beiden Seiten vorgetragen wurden, läbt kaum auf Aussöhnung hoffen. 
In den zwei folgenden Kapiteln geht es um die politische Situation in Preußen und die Beziehung Schleswig-Holsteins zu Dänemark. Holk stellt sich auf die Seite der Dänen. Seine spätere Berufung nach Kopenhagen ist damit vorbereitet.

Doch auch Christines Schicksalsweg wird weiterverfolgt. Ein kleines Lied von Waiblinger, das Elisabeth zum Abschluß des Abends singt, und dem Christine ergriffen lauscht, heißt "Der Kirchhof."

Fontane benutzt im wesentlichen vier verschiedene Methoden der Vordeutung und Vorahnung. Wir haben gesehen, wie Christine gefühlsmäßig stark auf alles reagiert, was ihr Leben und ihren bevorstehenden Tod bestimmt. Wehmütig denkt sie zurück an die Jahre des Glückes im alten Schloß. Sie ist "ergriffen" von dem Waiblinger Gedicht und so tief getroffen, daß sie sich aus der Gesellschaft zurückziehen muß. Das vorahnende Gefühl der Hauptperson ist also die erste Methode. Wir haben aber auch gesehen wie Fontane Dingsymbole heranzieht, um den Leser auf das Ende vorzubereiten. Das Schlos exinnert an einen "Tempel." Baupläne und Skizzen für eine "Familiengruft" werden entworfen und das Gedicht heißt "Der Kirchhof."

Im fünften Kapitel werden im Gespräch direkte Hinweise auf die Gefahr gegeben, in der sich Christine befindet. Arne und Schwarzkoppen unterhalten sich auf ihrer Heimfahrt über den Abend bei Familie Holk. Ganz offen besprechen sie die Unterschiede im Charakter der Eheleute. Sie fürchten, daß diese Unterschiede, wenn sich die Partner auf ihrer gegenwärtigen Haltung versteifen, schließlich zum Bruch führen werden. Arne bemerkt aber auch, dẩ Christine überempfindlich sei und nicht "ohne Schaden zu nehmen" (R.E., Bd. VI, S. 34) das Kirchhofslied hören könne. 
Die vierte Methode ist schlie3lich die direkte Aussage des Erzählers:

Trotz aller Liebe - seine leichtlebige Natur und ihre melancholische, sie stimmten nicht recht mehr zueinander, was ihr diese letzte Zeit, trotz alles Ankämpfens dagegen, mehr als einmal und leider in immer wachsendem Grade gezeigt hatte. (R.E., Bd. VI, S. 34 ).

Verfolgen wir weiter wie Fontane den Leser führt, so daß das Vorgeahnte schlieblich bewußt und von ihm erwartet wird.

Im sechsten Kapitel kommt ein Brief aus Kopenhagen an. Holk wird früher als erwartet zum Dienst an den Hof berufen. Dieser Brief veranläßt Christine, ihre Bedenken über Holks Dienstleistungen und das unbeherrschte Leben am Hof zu äußern. Holk meint aber, daß "das Gastmahl des seligen Belsazar" (R.E., Bd. VI, S. 48) noch nicht da.sei. Das Kapitel schließt wieder mit dem nun schon leitmotivisch auftretenden Gedicht "Der Kirchhof."

In den zwei folgenden Kapiteln werden die Vorbereitungen zu Holks Abreise getroffen. Schwager Arne übernimmt, wie schon früher, die Führung der Wirtschaft. Holk ist offensichtlich froh, daB er sich auf einige Zeit aus der bedrückenden Atmosphäre seines Heimes zurückziehen kann. Doch auch Christine "ertappt" sich bei einer ähnlichen Empfindung. In früheren Jahren hatten diese kurzen Trennungen beiden Eheleuten gut getan.

Wenn sie getrennt waren, waren sie sich innerlich am nächsten, denn es fielen dann nicht bloß die Meinungsverschiedenheiten und Schraubereien fort, sonder sie fanden sich auch wieder zu früherer Liebe zurück und schrieben sich zärtliche Briefe. (R.E., Bd. VI, S. 82).

Die Hoffnung, daß alles doch noch gut ausgehen werde, wird aber gleich wieder zerstört. Holk gibt seine Zustimmung zum Bau der Familiengruft.

Am folgenden Tage reist Holk nach Kopenhagen ab. Christine, die sich auf die stille Zeit gefreut hatte, ist aber bedrückt und ängstlich. 
In der vergangenen Nacht hat sie einen schwermütigen Traum gehabt, und obgleich sie von Traumdeutungen nichts hält, kann sie das "Bild" nicht loswerden. Sie erzählt der Dobschütz: "Ein Trauerzug war es, nur ich und du, und in der Ferne Holk. Und mit einem Male war es ein Hochzeitszug, in dem ich ging, und dann war es wieder ein Trauerzug." (R.E., Bd. VI, S. 94 ).

Der erste Trauerzug gilt der Scheidung. Die Hochzeit zeigt ihre Wiedervereinigung mit Holk, und der:zweite Trauerzug ist ihr Begräbnis. In diesem Traumbild hat Fontane das Ende des Romans unverhüllt dargestellt. Der Leser nimmt von Christine Abschied, die erst 20 Kapitel später wieder direkt in die Handlung einbezogen wird. Nur das letzte Wort des neunten Kapitels, "Tod," klingt nach und begleitet uns bis Christine wieder erscheint.

Das Mittelstück des Romans spielt in Kopenhagen. Wir sehen Holk in seiner Wohnung bei Frau Hansen. Die Tochter des Hauses hat groben. Eindruck auf ihn gemacht. Er ist sich seiner Gefühle nicht klar. Brigitte ist ihm "urheimlich." Doch sein Gedanke: "Ich darf ihrer in meinem Brief an Christine gar nicht erwähnen," (R.E., Bd. VI, S. 82), sagt mehr über ihn aus, als ihm selber bewußt ist. Er ahnt die Gefahr, in die diese Frau ihn bringen könnte. Der Leser fühlt sich in seiner Ahnung bestätigt, daß sich ein besseres Verhältnis zu Christine wohl doch nicht anbahnen wird.

Während des ersten Besuchs bei der Prinzessin, wird Holk Ebba von Rosenberg vorgestellt. Holk verneigt sich.mit "widerstreitenden Empfindungen." (R.E., Bd. VI, S. 94). Diese zwei Worte weisen schon auf das spätere Verhältnis zwischen Holk und Ebba hin. Es ist nur eine Frage 
der Zeit, bis sich Holk seiner Gefühle für Ebba bewuBt wird. Jetzt verstehen wir auch die Anspielung, die die Dobschütz bei der vermeintlichen Eifersucht Christines macht, "und wenn drüben in Kopenhagen auch jede dritte Frau die Frau Potiphar in Person wäre, du wärest seiner (Holks) doch sicher." (R.E., Bd. VI, S. 109).

Fin Gefühl der Sicherheit kommt aber weder bei Christine noch beim Leser auf. Das Gegenteil trifft eher zu. In einem Gespräch der Hofleute sagt die Prinzessin über ihr geliebtes Frederiksborg: "Ich habe nur immer erquickliche Ruhe hier gefunden, Ruhe, die weniger ist als Glück, aber auch mehr. Die Ruhe ist wohl das Beste." (R.E., Bd.VI, S. 109). Die letzten Worte ließen Holk aufhorchen. Es waren die gleichen Worte, mit denen das Waiblinger Lied begann, das Elisabeth Petersen gesungen hatte. "Das war noch keine Woche, und schon klang es ihm wie aus weiter, weiter Ferne." (R.E., Bd. VI, S. 109). Noch "keine Woche" und schon "weite, weite Ferne" weist deutlich auf die tiefe Gefühlstrennung der Eheleute hin, die nichts mit der kurzen zeitlichen Trennung zu tun hat.

Einen weiteren Hinweis auf das Verhältnis, das sich zwischen Holk und Ebba anbahnt und selbst schon auf den bevorstehenden Ehebruch, erhalten wir in dem Gespräch zwischen Holk und Brigitte. Sie sprechen über das Verhältnis des Königs zur Gräfin Danner. Es ist ein allgemein bekanntes Liebesverhältnis. Die Anspielung auf den "Schönheitsapfel" gilt weniger dem König als Holk, dem die ganze Sache seinen eigenen Worten nach "halb unverständlich" bleibt. Das "Unheimliche" das von Brigitte ausging, erkennt Folk jetzt als das "Verführerische." Seine Sinne sind ganz von ihrer Schönheit und ihrem Reiz befangen. Doch da 
er ihre Absicht erkennt, ist die Gefahr gemindert, und er setzt sich und schreibt an Christine.

Vor Brigitte ist er nun sicher. Ebba aber; die nicht so plump in ihrer Verführung ist, wird Erfolg haben. Wir hören von ihrer "Vergangenheit," die das Verhältnis zu Holk nicht nur möglich, sonder auch wahrscheinlich macht. Wir sehen, wie sie ganz offen mit Holk flirtet, und wie sich sein Widerstand ihr gegentiber verringert. Die Prinzessin versucht, Ebba von ihrem gefährlichen Spiel abzubringen. Ebba will aber nicht und meint: "Aber bin ich die richtige Adresse? Nun und nimmermehr. Holk ist es. Er ist seiner Frau Treue schuldig, nicht ich, und wenn er diese nicht hält, so kommt es auf inn und nicht auf mich." (R.E., Bd. VI, S. 142).

Noch einmal gibt uns Fontane für einen kurzen Augenblick Hoffnung, daß all unsere Befürchtungen nicht zutreffen werden. Holk erhält einen Brief von Christine und fühlt Liebe und Zuneigung in sich aufsteigen. Er vergleicht Christine mit Brigitte und Ebba:

Sie war doch die Beste. Was bedeutete daneben die schöne Brigitte? Ja, was bedeutete daneben selbst Ebba? Ebba war eine Rakete, die man solange sie stieg, mit einem staunenden "Ah" begleitete, dann aber war's wieder, vorbei, schließlich doch alles nur Feuerwerk, alles künstlich; Christine dagegen war wie das einfache Licht des Tages. (R.E., Bd. VI, S. 145).

Dieses "einfache Licht des Tages" kann sich aber nicht mehr gegen die "steigende Rakete" und das "Feuerwerk" durchsetzen. Der Kontrast von Tag und Nacht zeigt deutlich, auf welchem Wege sich Holk befindet. Beim zweiten Lesen des Briefes schwindet dann auch das Gefühl der Liebe und was bleibt ist: "das Schrecknis ihrer (Christines) Vorzüglichkeit." (R.E., Bd. VI, S. 146). 
Von diesem Augenblick an gibt es kein Zurück mehr. Nicht nur der krasse Umschwung von Holks Gefühlen zeigt es, sondern auch die Verlagerung der Handlung an einen entlegenen Ort. Die Prinzessin will die Weihnachtstage in Frederiksborg verbringen. Deshalb verläßt man Kopenhagen. Doch bevor die Reisegesellschaft SchloB Frederiksborg erreicht hat, hören wir, daß es zwar ein "gutes Schloß" sei, ohne "Blut und Mord" doch nicht "ohne Schuld und Sünde." (R.E., Bd. VI, S. 151). Später, in der Halle von Frederiksborg, finden wir die Gesellschaft vor dem Kamin. Verschiedene Gemälde liefern den Gesprächsstoff. Das Bildnis von Brigitte Goja gibt der Prinzessin dann noch einmal Anlaß, Ebba zu warnen. Brigitte Goja wurde von einem katholischen Bischof umworben. Doch es kam nicht zur Ehe. Sie heiratete Herluf Trolle. "Da tat sie recht. Nicht wahr, Ebba?" (R.E., Bd. VI, S. 163) meint die Prinzessin. Ebba legt sich nicht fest und antwortet nur mit: "Vielleicht,...vielleicht auch nicht." (R.E., Bd. VI, S. 163). Wieder ist es ein Traum, der das künftige Geschehen verbildlicht. In der ersten Nacht in Frederiksborg träumt Holk, angeregt durch das Gemälde einer Seeschlacht, er wäre mit dem Schiff "in die Luft geflogen, und als er ein Stück Mast gepackt hatte, um sich daran zu retten, war Ebba von der anderen Seite her, ganz wie ein lheerweib, aufgetaucht und hatte ihn von dem Mast fort- und in die Flut zurückgerissen." (R.E., Bd. VI, S. 163). Holk wird auch wirklich versuchen, sich zu retten, aber Ebba wird inn doch mit sich reißen.

Am nächsten Tag bei einem Spaziergang bewundern Holk und Ebba die Lage von SchloB Frederiksborg. Holk kritisiert die Bauweise und meint:

Die zwei Backsteintürme, drin wir wohnen, die hätten mit ihrem prächtigen Rot etwas höher hinaufgeführt werden müssen, 
und dann erst die Schiefer- oder Schindelspitze. Jetzt sieht es aus, als solle man aus der untersten Turmluke gleich auf das große Schrägdach hinaustreten, um draußen an der Dachrinne hin, eine Promenade zu machen. (R.E., Bd. VI, S. 172).

Wie wir sehen werden, verdanken Holk und Ebba dieser genauen Beobachtung des Schloßbbaus ihr Ieben. Als einige Wochen nach diesem Spaziergang das Schloß in Flammen aufgeht, entkommen sie über das Dach.

Doch bevor wir zu dieser bedeutenden Stelle des Schloßbrandes kommen, sollen noch einige weitere Begebenheiten erwähnt werden. Wie gewöhnlich findet auch an diesem Tage bei Tisch ein anregendes Gespräch statt. Holk und Ebba vertreten den entgegengesetzten Standpunkt in der Frage "was die Menschen eigentlich interessiere." Ebba meint, es wären die kleinen täglichen Ereignisse, während Holk den "privaten Standpunkt" ablehnt und nur den "historischen" großen Stils gelten lassen will. Ebba antwortet ihm:

Großer Stil: Bah, ich weiß wohl, die Menschen sollen tugendhaft sein, aber sie sind es nicht, und da, wo man sich darin ergibt, sieht es im ganzen genommen besser aus als da, wo man die Moral bloß zur Schau stellt. Leichtes Leben verdirbt die Sitten, aber die Tugendkomödie verdirbt den ganzen Menschen. (R.E., Ba. VI, S. 179).

Und wie um das eben Gesagte noch zu bekräftigen, fällt ein Wachsengel aus dem geschmückten Weihnachtsbaum. Pentz interpretiert dieses Zeichen auch gleich richtig als "gefallenen Engel" und fragt: "Wer es wohl sein mag?" (R.E., Bd. VI, S. 180). Diese Frage bleibt zunächst unbeantwortet. Die Vermutung aber, daß Holk der "gefallene Engel" sei, bekräftigt sich in der Eislaufszene. Ebba und er hatten die kleine Ausflugsgesellschaft zurückgelassen und liefen über das Eis der "letzten Sicherheitsgrenze" zu. Doch im letzten Augenblick bog Holk ab und ri:B Ebba mit sich. Diese "romantische Eskapade" wird am Abend von allen Anwesenden ausgiebig besprochen und ausgesponnen. Das Thema ist 
"Leidenschaft" und deutet auf Holks Gemütszustand hin. In der gleichen Nacht unterliegt er seiner Leidenschaft und begeht den Ehebruch, der sich seit seiner Bekanntschaft mit Ebba angebahnt hatte. Der Brand, der in dieser Nacht ausbricht und den Teil des Schlosses vernichtet, in dem Holk und Ebba untergebracht waren, bringt ihr Verhältnis ins Licht. Holk rettet sich und Ebba, weil er sich an die Konstruktion des Turmund Schlobdaches erinnerte. In dieser Rettung sieht er aber auch ein Zeichen, daB sein Verhältnis zu Ebba gut ist. Er meint: "Wäre der Weg, den mein Herz all diese Zeit ging, ein falscher gewesen, so hätte mich die Strafe getroffen, mich und Ebba, und wir wären ohnmächtig zusammengesunken und erstickt und hätten uns nicht in die Luft und Freiheit hinaus gerettet. (R.E., Bd. VI, S. 217).

So sicher er dieser Auffassung schien, beschlichen ihn doch Zweifel. Es gelang inm aber, die Zweifel zu unterdrücken, da er die Situation nicht objektiv, sondern seinen eigenen wünschen gemäß betrachtete. Er überzeugte sein Gewissen davon, daß er sich von Christine trennen müsse, und daß er nur an Ebbas Seite das wahre Glück finden könne. Ebbas war er sich sicher, und die Zustimmung der Prinzessin schien ihm auch gewiB. Er bat un Urlaub und kehrte nach SchloB Holkenäs zurück. Wieder befielen ihn Zweifel. Doch der Gedanke an Ebba, daß sie ihn auslachen und sich von ihm abwenden würde, gab ihm Mut. Christine war auf das bevorstehende Zusammentreffen mit Holk vorbereitet. Sie hatte aus seinen wenigen Briefen entnommen, was er ihr jetzt zu sagen gekommen war. Nur über die Frage, "ob Brigitte oder Ebba," war sie unsicher. Doch die Antwort darauf war unwichtig. Bevor sie aber geht, gibt sie Holk zu verstehen, daß er kein Glück finden und "auf ein unglückliches Leben zurückblicken" wird. 
Nach Kopenhagen zurückgekehrt, erfährt Holk von Ebba, daß für sie die ganze Geschichte "ein bloßes Spiel" war, das sie aus Langerweile gespielt hatte. Für den Leser kommt Ebbas Ablehnung nicht als überraschung, wie für Holk. Holk selbst sah Ebba am Anfang, wie sie wirklich war: geistreich, verspielt und auf ihren eigenen Vorteil bedacht. Doch sie verstand es, ihn zu bezaubern, und er verfiel ihr. Seine eigene Leidenschaft hat ihm dann völlig den klaren Blick genommen, und da er seine wïnsche auch für die ihrigen hielt, glaubte er sich seines Glückes versichert. Auf die Ablehnung war er nicht vorbereitet. Selbst in diesem Augenblick glaubte er noch, daß sie ihre Meinung geändert hatte, aus welchen Gründen auch immer. Für Holk bleibt jetzt weiter nichts, als sich von Kopenhagen zurückzuziehen.

Anderthalb Jahre hält sich Holk im Ausland auf, ohne Glück und Frieden zu finden. Als sich nach Ebbas Heirat und durch die Vermittlung von Arne und Schwarzkoppen die Gelegenheit zu einer Wiederheirat mit Christine bietet, kehrt Holk nach Holkenäs zurück. Am Johannistag findet die Trauung unter Teilnahme der Freunde und Dorfbewohner statt, und man trinkt auf das "Glück von Holkenäs." Doch der Erzähler gibt zu verstehen: "... eine rechte Freude wollte nicht laut werden, und jedem Anwesenden kam ein banges Gefühl davon, daỉ man das "Glück von Holkenäs," wenn es überhaupt da war, nur heute noch in Händen hielt, um es vielleicht morgen schon zu begraben." (R.E., Bd. VI, S. 255). Das "bange Gefühl" bestätigt sich einige Monate später. So schmerzlich Christines Freitod für ihren Mann und ihre Freunde war, so wenig unerwartet kam er. Für den aufmerksamen Leser war es eine Frage der Zeit.

Wir haben gesehen wie Fontane durch die verschiedenen Mittel der Vordeutung jedes Ereignis vorbereitet hat. Auf den ersten Seiten des 
Romans hat er die Grundsituation der Holkschen Ehe klar umrissen und den Konflikt dargestellt. Die weitere Entwicklung des Konflikts hat er stufenweise enthüllt und die Ahnungen des Lesers bestätigt, so daß das tragische Ende erwartet wurde und folgerichtig eintreffen mubte.

HOLK UND CHRISTINE:

EINE STUDIE ZUR VERSCHIEDENHEIT DER CHARAKTERE

Die gleiche Sorgfalt, mit der Fontane die Ereignisse des Romans entwickelte, verwendet er auch in der Darstellung der Hauptpersonen. Der Erzähler vermittelt am Anfang des Romans die Kenntnis seiner Personen in einer Skizze und läßt den Leser die Weiterentwicklung selbst verfolgen. Der. Konflikt zwischen den Eheleuten wird aus der "Verschiedenheiten der Charaktere" hergeleitet: "Holk, so gut und vortrefflich er war, war doch nur durchschnittsmäßig ausgestattet und stand hinter seiner Frau, die sich höherer Eigenschaften erfreute, um ein beträchtliches zurück." (R.E., Bd. VI, S. 12). Dieser Unterschied bestand schon vor ihrer. Ehe. Daß er sich nicht negativ auf das Verhältnis der Eheleute, in den ersten Jahren auswirkte, wird folgendermaßen erklärt: Holk und Christine hatten aus Liebe geheiratet. Ihre Liebe überwand die Gegensätze. Die Geburt ihrer arei Kinder und der Tod des jüngsten steigerte noch das Gefühl ihrer Zusammengehörigkeit. Erst als die Kinder herangewachsen waren und die Eheleute mehr Zeit für sich hatten, machten sich die Gegensätze in ihren Charakteren bemerkbar. Beide Partner wurden sich dieser Gegensätze bewußt. Holk litt unter Christines "Tugenden" und wïnschte sich eine "weniger vorz̈̈liche Frau." Christine litt unter Holks "Mittelmäßigkeit" und wünschte sich einen Mann, der feste Standpunkte vertrat und sich nicht vom 
Augenblick treiben ließ. Selbst wenn sein Standpunkt ihrem entgegengesetzt wäre, wäre es besser, als keinen Standpunkt zu haben. In der Frage der Religion äußert sich Christine folgendermaßen über Holk zu. ihrem Bruder Arne:

Ein Freigeist sein, das kann er nicht, dazu reichen seine Gaben nicht aus, auch nicht die seines Charakters. Und das ist eben das Schlimme. Mit einem Atheisten könnte ich leben, wenigstens halte ich es für möglich, ja, mehr, es könnte einen Reiz für mich haben, ernste Kämpfe mit ihm zu bestehen. Aber davon ist Helmuth weit ab. Ernste Kümpfe! Das kennt er nicht. (R.E., Bd. VI, S. 61):

Fontane zeigt keine Charakterentwicklung. So wie Christine am Anfang des Romans ist, so ist sie auch bis zum Ende. Das gleiche gilt für Holk.

Christine wurde im Herrnhuter Pensionat erzogen. Religion und Frömmigkeit formten ihren Charakter und wurden für ihren Lebensweg bestimmend und richtungsweisend. Sie sieht das diesseitige Leben als Bewährung; als Vorḅereitung auf das Jenseits. Dazu gehört die strenge Pflichterfüllung. Die eigenen Wünsche müssen zu Gunsten der Pflicht zurücktreten. In der Erziehungsfrage drückt sie es Holk gegenüber aus: "Du bedarfst der Kinder, sagst du. Glaubst du, daß ich ihrer nicht. bedarf... Aber das Glück meiner Kinder gibt mir mehr als mein Behagen, und das, was die Pflicht vorschreibt, fragt nicht nach Wohlbefinden." (R.E., Bd. VI, S. 43).

Christine sieht das Leben vorwiegend von der unerbittlich ernsten Seite. Nicht das persönliche Glück ist das wichtigste, sondern "sich bezwingen" und die Anforderungen des Lebens willig auf sich nehmen. Da diese Anforderungen oft hart sind, sehnt sich.Christine nach Frieden und Ruhe, erkennt aber, daß sie erst im Tode die gewïnschte Ruhe finden wird. So ist ihr Leben von Todesahnung und Todessehnsucht überschattet. 
Lebensmüdigkeit spricht aus ihrer ganzen Haltung. Ihr fehlt die Sorglosigkeit, die ihr helfen könnte, von ihrer Prinzipienstrenge loszukommen. Sie ist aber auch nicht fähig, Hilfe von außen anzunehmen, denn, wie Schwarzkoppen richtig feststellt, gehört sie zu denen, die "keine Konzessionen machen und durch Starrheit und Unerbittlichkeit sich eine Rüstung anzulegen wissen, die besser schließt als die Rüstung eines milden und liebevollen Glaubens." (R.E., Bd. VI, S. 39).

Christine leidet unter ihrer eigenen Unerbittlichkeit. Sie weiB, daß3 sie das Leben schwer nimmt und anderen das Ieben schwer macht. Sie versucht auch, sich zu ändern, aber es gelingt ihr nicht. DaB sie es versucht, macht sie sympatisch und zeigt die ganze Tragik ihrer Situation. "Schuld ist überall, und vielleicht ist meine die größere... GewiB, ich klage dich oft an und will es nicht leugnen, aber ich darf auch sagen, ich verklage mich vor mir selber." (R.E., Bd. VI, S. 50). Mit diesen Worten hat sie ihren inneren Zwiespalt ausgedrückt. Sie bemüht sich um einen Kompromiß, findet ihn aber nicht. Was bleibt ist Resignation.

Holk ist in vielem das genaue Gegenteil seiner Frau. Er hat keine Ideale, keine Prinzipien. Religion und Erziehung steht er gleichgültig gegenüber. An Bildung und Intelligenz ist er Christine unterlegen. All "seine liebenswürdigen Eigenschaften," wie Weltfreude, Anpassungsfähigkeit, Charm und Humor machen ihn viel lebensfähiger als seine ernste Gattin, reichen aber nicht aus, un das Glück seiner Ehe zu sichern. Denn beide Ehepartner verstehen es nicht, Kompromisse zu schließen. Christines Rechthaberei, ihr festes Bestehen auf ihrem Standpunkt, ihre konservative Weltanschauung treffen immer wieder auf Holks Unentschlossenheit. Anfänglich bewunderte Holk die "Tugenden" 
seiner Frau, ihre Schönheit, Klugheit und vor allem Frömmigkeit, aber jetzt sieht er diese Tugenden nur noch als "schreckliche Vorzüglichkeiten." Doch genau so wenig wie er zu Christine paßt, paßt er zu den Hofleuten in Kopenhagen. Christine kennt ihn genau und sagt zur Dobschütz" "Er ist gut unò treu, der beste Mann von der Welt, das ist richtig, aber doch auch schwach und eitel, und Kopenhagen ist nicht der Ort, einen schwachen Charakter fest zu machen." (R.E., Bd. VI, S. 70). In Kopenhagen wird von Ebba die gleiche Beobachtung ausgesprochen. Sie erkennt sein "gutes schwaches Herz," urteilt aber viel härter über seinen Charakter als Christine:

Sein Charakter ist das recht eigentlich Schwache an ihm. Und was das schlimmste ist, er weiB es nicht einmal....Er ist moralisch, ja beinah tugendhaft und schielt doch begehrlich nach der Lebemannschaft hinüber. Und diese Halbheit ist die schlimmste. (R.E., Bd. VI, S. 141).

Christine fürchtet, dafi Holk aus Schwäche der Verführung unterliegen könnte; Ebba aber meint, daß er die Verführung herbeiwünscht.

Die "Halbheit," von der Ebba sprach, erkennt auch Christine als den Grundfehler seines Charakters. Sie willigt in die Scheidung ein, weil sie nicht "für halbe Verhältnisse" sei. Doch Holk kann die "Halbheit" nicht überwinden. Er ringt sich nie zu einer klaren Entscheidung durch. Die Umstände, in denen er sich jeweils befindet, treffen die Entscheidung für ihn. Im zweiten Kapitel sehen wir Holk als einen begeisterten Landwirt und im sechsten als einen ebenso begeisterten Kammerherrn, der es zufrieden ist, die Landwirtschaft seinem Schwager zu überlassen. Den gleichen Gesinnungswechsel zeigt er in der Frage der Erziehung. Holk will nicht, daß seine Kinder im Pensionat erzogen werden, willigt aber sofort ein, als er nach Kopenhagen berufen wird. Er ist, wie Schwarzkoppen treffend bemerkt, ein "Augenblicksmensch 
und hält zu dem alten Trost: Nach uns die Sindflut." (R.E., Bd. VI, S. 36). Der Augenblick bestimmt seine jeweilige Weltanschauung und Lebensführung. Seine Tochter Asta bestätigt die Verantwortungslosigkeit ihres Vaters, wenn sie auf die Frage ihrer Freundin, welchen der Eltern sie bevorzuge, antwortet: "Die Mama bedrückt uns oft, aber sie sorgt doch auch für uns, und der Papa erfreut uns jeden Augenblick, aber im Ganzen kümmert er sich nicht recht um uns. Er ist mit seinen Gedanken immer woanders und die Mama immer bei uns." (R.E., Bd. VI, S. 56). So ist es auch weiter nicht verwunderlich, daB Holk in Kopenhagen seine Familie fast vergibt und nur aus Pflicht einige Briefe nach Hause schreibt. Arnes Vorwurf trifft ihn zu recht: "Für Deine Frau, Deine Kinder hast Du während dieser langen Zeit keine zwanzig Zeilen gehabt, immer nur Fragen, denen man abfühlte, daß sie nach Antwort nicht sonderlich begierig waren." (R.E., Bd. VI, S. 187). Holk ist ganz betroffen und überrascht von den Anschuldigungen, die sein Schwager gegen ihn vorbringt. Genau so überrascht war er, als Ebba seinen Heiratsantrag ablehnte. Er lebt dem Augenblick und sieht die Zusammenhänge nicht. Er ist, wie Arne ihn richtig nannte, ein "Vogel StrauB," der den Kopf in den "Busch" steckt und "die Gefahr nicht sehen" will. (R.E., Bd. VI, S. 28).

Fontane zeigt, wie Holk aus Unüberlegtheit und Leichtsinn schuldig wird. Es ist nichts Bösartiges in seinem Handeln. Er läBt sich leicht von seiner Umgebung beeinflußen, und wenn ihn sein gutes Gewissen warnt, versucht er es zu beruhigen. Doch das gelingt ihm fast nie. In seinem Antwortsbrief an Arne versucht er die Vorwürfe zu widerlegen. Er scheint sich von seiner Unschuld überzeugt zu haben. Beim Überlesen des Briefes aber spührt er, daß "diè Befriedigung darüber hinschwand und ihn nichts 
mehr daraus ansah als das Bekenntnis seiner Schuld." (R.E., Bd. VI, S. 195). Sein Schuldgefühl hält aber nie lange an. So begeht er fast unbewust im Augenblick der Leidenschaft den Ehebruch. Er rechtfertigt diesen Fehltriti, indem er Christine die Schuld zuschiebt:

Christine hat mich von sich wegerkältet. Ja, das ist das rechte Wort, und solche sichmehrende Kälte, das ist schlimmer als Streiten und Heftigsein. Eine Frau soll eine Temperatur haben, ein Temperament und Leben und Sinne. Aber was soll ich mit einem Eisberg? Und wenn er das klarste Eis hat, das klarste ist gerade das kälteste, und ich will nicht erfrieren. (R.E., Bd. VI, S. 223).

Es geht Holk also nur um sein eigenes Glück, und diesem Egoismus opfert er seine Ehe. Doch Fontane zeigt, dab aus Schuld kein Glück entstehen kann. Ebba weist ihn $a b$, und seine zweite Ehe mit Christine bringt das verlorene Glück nicht zurück.

Während Christine im Freitod die ersehnte Ruhe findet, bleibt Holk diese Ruhe versagt. Am Ende erhebt er keinen Anspruch auf Glück: "Denn ein Unrecht fordert nicht bloß seine Sühne, sondern diese Sühne befriedigt uns auch, weil sie unserem Rechtsgefühl entspricht." (R.E., Bd. VI, S. 257). Holk akzeptiert seine Strafe, doch für sein Mitverschulden an Christines Tod findet er keine Sühne. 
KAPITEL II

VORGESCHICHTE ZU EFFI BRIEST

In seinem Roman Effi Briest hat Theodor Fontane das Schicksal einer jungen Frau und den moralischen Zerfall ihrer the dargestellt. Wie die meisten der Fontaneschen Romane beruht auch dieser auf einer wahren Begebenheit. Fontane kannte Baron Ardenne, und als er fragte: "Wo ist denn jetzt Baron A.?" 8 teilte ihm Frau Lessing die Geschichte Elisabeths una Armands von Ardenne mit.

Ardenne war musikalisch begabt. Er zeichnete unđ̄ kannte sich auch in der Literatur aus. In Berlin gehörte er dem Großen Generalstab an und ging ganz in seinem Dienst auf. Elisabeth litt unter der Unaufmerksamkeit ihres Mannes. Als das Ehepaar in die Nähe von Düsseldorf versetzt wurde, gehörte zu ihrem dortigen Freundeskreis der Amtsrichter und Maler Emil Hartwick. Hartwicks Ehe war auch nicht glücklich und Elisabeth und Hartwick beschlossen, sich von ihren Ehegatten scheiden zu lassen und einander zu heiraten. Ardenne erfuhr aus Hartwicks Briefen an seine Frau von diesem Plan und reichte die Scheidungsklage ein. Gleichzeitig forderte er Hartwick zum Duell. Hartwick wurde schwer verwundet und starb am 1. Dezèmber, 1886. Im März des folgenden Jahres wurde die Ardennesche Ehe geschieden. (R.E., Bd. VI, S. 527).

In einem Brief an Hans Hertz vom 2. März, 1895, äußerte sich Fontane ausführlich über den Eindruck, den die Geschichte der Ardennes ouf ihn gemacht hatte: 
Meine Gönnerin Lessing...erzühlte mir auf meine Frage: 'Was macht denn der?' (ein Offizier, der früher viel bei Lessings verkehrte und den ich nachher in Innstetten transponiert habe), die ganze Effi-Briest-Geschichte, und als die Stelle kam, 2. Kapitel, wo die spielenden Mëdchen durchs Weinlaub in den Saal hineinrufen: 'Effi, komm,' stand mir fest: 'Das mubt du schreiben.' (F.Br., Bd.2, S. 368).

Die gleiche Episode berichtet Fontane auch in seinem Brief an Spielhagen vom 21. Februar 1896:

Die ganze Geschichte ist eine Ehebruchsgeschichte wie hundert andere mehr und hatte, als mir Frau L. davon erzählte, weiter keinen großen Eindruck auf mich gemacht, wenn nicht (vergl. das kurze 2. Kapitel) die Szene bez. die Worte: 'Effi, komm,' darin vorgekommen wären. Das Auftauchen der Mädchen an den mit Wein überwachsenen Fenstern, die Rotköpfe, der Zuruf und dann das Niederducken und Verschwinden machten solchen Eindruck auf mich, daw aus dieser Szene die ganze lange Geschichte entstanden ist. An dieser einen Szene können auch Baron A. und die Dame erkennen, daß ihre Geschichte den Stoff gab. (F.Br., Bd. 2, S. 368).

Die gehörte "Geschichte" liegt also als Handlungsskelett dem Roman zugrunde. Den Anlab für den Roman aber sah Fontane in der oben erwähnten Szene und in den Worten "Effi, komm." Während Armand von Ardenne für Innstetten Model stand, hat Fontane für seine Effi nicht Elisabeth von Ardenne als Vorbild genommen, sondern ein fünfzehnjähriges Mädchen, das er zufällig sah:

Das Mädchen war genau so gekleidet, wie ich Effi in den allerersten und dann auch wieder in den allerletzten Kapiteln geschildert habe: Hänger, blau und weiB gestreifter Kattun, Ledergürtel und Matrosenkragen. Ich glaube, daß ich für meine Heldin keine bessere Erscheinung und Einkleidung finden konnte...das Schicksal schickte mir die kleine Methodistin. (F.Br., Bd. 2, S. 368).

Die äuĐere Erscheinung dieser "kleinen Methodistin" hat Fontane zwar für seine Effi entliehen, ihr Wesen aber ist ganz seine Schöpfung. 
BESPRECHUNG VON EFFI BRIEST

UNTER DEM GESICHTSPUNKT DER VORDEUTUNG

Theodor Fontane betonte wiederholt die Bedeutung der kleinen Szene am weinberankten Fenster, die den Zuruf "Effi, komm" enthält, für die Entstehung des Romans. Auf den ersten Blick erkennt der Leser nicht, dab hier der Ausgangspunkt für das weitere Geschehen liegt. Erst rückblickend, als Vater Briest Effi mit den gleichen Worten in das Elternhaus zurückruft, wird deutlich, daß diese Szene einen Wendepunkt in Effis Leben darstellt.

In den beiden einführenden Kapiteln sehen wir Effi beim Spiel mit ihren Freundinnen. Der weite Garten, das sonnenbeschienene Haus und die übermütige Stimmung der jungen Mädchen zeugen von einem unbeschwerten Leben. Doch in den Gesprächen klingen Miltöne an, die die friedliche Stimmung stören, Effi erzählt eine "Liebesgeschichte mit Entsagung." Es ist die Geschichte ihrer Mutter und Baron Innstettens, der zu jung war, sie zu heiraten. Wir hören auch von dem türkischen Brauch, untreue junge Frauen zu ertränken.

Das Spiel der Mädchen wird durch den Besuch Innstettens unterbrochen, der gekommen ist, um um Effis Hand anzuhalten:

Effi, als sie seiner ansichtig wurde, kam in ein nervöses Zittern; aber nicht auf lange, denn im selben Augenblick fast, wo sich Innstetten unter freundlicher Verneigung inr näherte, werden an dem mittleren der weit offenstehenden und von wildem Wein halb überwachsenen Fenstern die rotblonden Köpfe der Zwillinge sichtbar, und Herthe, die Ausgelassenste, rief in den Saal hinein: "Effi, komm." (R.E., Bd. VII, S. 19).

Effis "Zittern" steht so ganz im Gegensatz zu allem, was ihre vorherige Haltung auszeichnete. Ihre Sicherheit hat sie verlassen und es scheint, als hütte sie Angst vor Innstetten. Doch diese Angst 
verfliegt sofort, als sie in ihre wohlvertraute Welt zurückgerufen wird. Innstetten dagegen kann von dem Eindruck, "als wäre der kleine Hergang doch mehr als ein blober Zufall gewesen" (R.E., Bd. VII, S. 22), nicht loskommen. Dieser Eindruck äberträgt sich auch auf den Leser und bestätigt in ihm das unangenehme Gefühl, daß diese the nicht glücklich verlaufen wird.

Während der kurzen Verlobungszeit zeigt Effi wenig Teilnahme an den Hochzeitsvorbereitungen, die von ihrer Mutter getroffen werden. Sie ist mit allem zufrieden und freut sich nur über die Abwechslung, die die geschäftigen Wochen mit sich bringen. Das letzte Gespräch zwischen Mutter und Tochter vor der Hochzeit drückt wieder die Zweifel aus, die Effis und Innstettens Verbindung hervorgerufen hat. Es ist nicht so sehr der Altersunterschied, der einer glücklichen the im Wege steht, es ist viel mehr ihre verschiedene Wesensart, wie Effi richtig fühlt. Sie sagt zu ihrer Mutter, Innstetten sei ein Mann von "Charakter," von "Grundsätzen": "Ach, und ich...ich habe keine. Sieh, Mama, da liegt etwas, was mich quält und ängstigt. Er ist so lieb und gut gegen mich und so nachsichtig, aber...ich fürchte mich vor ihm." (R.E.,.Bd.VII, S. 36). Effis Worte machen einen tiefen Eindruck auf die Mutter. Sie, die nur die Vorteile dieser Verbindung gesehen hatte, wird unsicher. Im Gespräch mit ihrem Mann nach der Hochzeit gibt sie ihren Befürchtungen Ausdruck: "Sie (Effi) gehört zu denen, die so recht eigentlich auf Liebe gestellt sind...Wohl möglich, daß es alles mal kommt, Gott verhüte es, aber noch ist es nicht da." (R.E., Bd. VII, S. 41). Was da ist, ist "Vergnügungssucht und Ehrgeiz." Effis Ehrgeiz wird Innstetten befriedigen, denn er ist ein "Karrieremacher." 
Für die stündliche kleine Zerstreuung und Anregung (meint Frau Briest), dafür wird Innstetten sehr schlecht sorgen... Das wird eine Weile so gehen ohne viel Schaden anzurichten, aber zuletzt wird sie's merken, und dann wird es sie beleidigen. Und darn weis ich nicht, was geschieht. Denn so weich und nachgiebig sie ist, sie hat auch etwas Rabiates und läßt es auf alles ankommen. (R.E., Bd. VII, S. 42).

Nach der Hochzeitsreise läßt sich das Ehepaar von Innstetten in Kessin nieder, wo Innstetten Landrat ist. Effi ist beglückt, eine so bunte Stadtbevölkerung vorzufinden. Gleichzeitig aber beschleicht sie ein Gefühl von Angst und Unsicherheit in dieser fremden Ungebung. Bei den Adelsfamilien der näheren Ortschaften findet Effi meist kritische Aufnahme. Sie selbst ist von ihnen nicht sonderlich beeindruckt und meint, es wären "mittelmäßige Menschen, von meist zweifelhafter Liebenswürdigkeit." (R.E., Bd. VII, S. 68). Sie hat kein Verlangen, mit ihnen nähere Beziehungen aufzunehmen und entschließt sich zum "Einsiedlertum." Nur den Apotheker Gieshübler, der fllr sie "der einzige richtige Mensch" in Kessin ist, sieht Effi gern.

Die ersten Wochen in Effis neuer Heimat waren recht abwechslungsreich gewesen. Als aber der Alltag einsetzte, und Innstetten sich mehr und mehr seinen Pflichten widmen multe, wozu auch längere Besuche beim Fürsten zählten, überkommt Effi Heimweh nach Hohen-Cremmen. Sie zweifelt an ihrer Position und meint, daß sie sich nicht zur "großen Dame" eigne. Zu ihrer Unsicherheit und ihren Zweifeln gesellt sich eine unaussprechliche Angst vor dem Chinesenspuk, der in ihrem Hause umgeht. Innstetten kann sie zwar wieder beruhigen, aber als tffi ihn bittet, ein anderes Haus $\mathrm{zu}$ beziehen, kommt es $\mathrm{zu}$ einem kleinen Streit zwischen den Eheleuten. Effi muss sich fïgen, denn ein Umzug wegen des Chinesenspuks wïrde Innstetten der "Lücherlichkeit" preisgeben, und er meint: "Von solcher Lächerlichkeit kann man sich nie wieder erholen." (R.E., Bd. VII, s. 83$)$. 
Effis Einsamkeit wird durch das Gefühl, von ihrem Mann nicht recht verstanden zu werden, nur noch größer. Doch Gieshüblers Einladung zu einem Musikabend läßt sie ihren Kummer vergessen. Sie freut sich über die Abwechslung, und in Gesellschaft interessanter Menschen fühlt sie sich wieder ganz wohl. Neue Hoffnung schöpft sie auch aus der Tatsache, daß sie ein Kind erwartet und schreibt ihrer Mutter: "Wie glücklich ich selber im Hinblick darauf bin, brauche ich nicht erst zu versichern, schon weil ich dann Leben und Zerstreuung um mich her haben werde." (R.E., Bd. VII, S. 103). Die Geburt ihres Kindes soll ihr aber auch eine Rückkehr ins Elternhaus bringen, und so wartet sie sehnsüchtig auf den Sommer.

Auch die Ankunft des neuen Landwehrbezirkskommandeurs hätte ihr in ihrem "gesellschaftlichen Notstand" helfen können, besonders da Innstetten und Major von Crampas schon während des Krieges in derselben Brigade waren. Doch Frau von Crampas' Eifersucht verhindert freundschaftlichere Beziehungen zwischen den beiden Familien.

Wenige Wochen vor der Geburt des Kindes führt ein glücklicher Zufall Roswitha als Kindermädchen ins Haus. Effi faßt gleich Zutrauen zu ihr. Roswithas Gegenwart vertreibt alle Angst.

Kurz nach der Taufe der kleinen Annie reist Effi nach HohenCremmen, wo sie sechs Wochen "voller Glück und Zufriedenheit" verbringt. Nur in Gesprächen mit dem Vater klingen Bedenken über Effis Eheglück an. Sie bemüht sich, diese Bedenken zu zerstreuen, muß aber immer wieder erkennen, $d a B$ sie in ihrer the nicht glücklich ist.

Als Effi nach Kessin zurückgekehrt war, bemerkt Innstetten, daß sie sich verändert hat. Das "Kind" hat sich in eine "verführerische" junge Frau verwandelt. Effi freut sich dieser Beobachtung, und alles 
weist auf glücklichere Tage für das Ehepaar, Major von Crampas gesellt sich jetzt öfters zu den Innstettens. Alle drei unternehmen gemeinsame Ausritte an den Strand. Bald aber kann sich der Baron nicht mehr an den Ausflügen beteiligen, denn die Wahlkampagne nimmt seine Zeit in Anspruch. Effi und Crampas nutzen aber das schöne Wetter zu weiteren Ritten. Ihre Gespräche werden intimer, und als das winterwetter alledem ein Ende macht, freut sich Effi, denn, obwohl sie sich nichts hatte zu Schulden kommen lassen, "hatte sie ganz leise das Gefühl einer überstandenen Gefahr." (R.E., Bd. VII, S. 149). Überstanden ist die Gefahr aber nicht. Sie hat noch gar nicht begonnen. Immer öfters schweifen Effis Gedanken zu Crampas. Sie fühlt sich bedrückt und leidet unter seinem Fernbleiben. "Eigentlich war es ihr lieb, aber auch wieder nicht, seine Huldigungen erfüllten sie mit einem gewissen Bangen, und seine Gleichgültigkeiten verstimmten sie; sie sah ein, es war nicht alles so, wie's sein sollte." (R.E., Bd. VII, S. 155). Doch diese Einsicht kommt zu spät. Effi kann ihrem Drang nach Abenteuer und Zerstreuung nicht widerstehen. Sie ist hilflos gegen Crampas. In dem Sinne äuBert sie sich zu Gieshübler, ohne wirklich zu wissen, was sie sagt. "Der Major hat so was Gewaltsames, er nimmt einem die Dinge gern über den Kopf fort. Und man muis dann spielen wie er will, und nicht, wie man selber will." (R.E., Bd. VII, S. 151). Gegen ihren Willen, aber nicht. gegen ihre Wünche, verwickelt sie sich mehr und mehr in das "Verbotene, das Geheimnisvolle" (R.E., Bd. VII, S. 177) unter dessen Macht sie steht. Innstettens unerwartete Versetzung nach BerIin erscheint ihr als Befreiung aus ihrer Situation und ihrer Schuld. Ein zweiter glücklicher Zufall bringt einen Brief von Frau Briest aus Berlin, in dem sie sich anbietet, Effi bei der Wohnungssuche behilflich zu sein. In 
wenigen Tagen trifft Effi ihre Reisevorbereitungen und ist entschlossen, nicht nach Kessin zurückzukehren. Einige Wochen später trifft auch Innstetten in Berlin ein, und für beide Eheleute kommen glückliche Zeiten. Doch hin und wieder tauchen vor Effis Augen Bilder aus der Kessiner Zeit auf, und die Angst verläßt sie nie ganz, daß ihr jetziges Glück doch noch zerstört werden könnte.

So vergingen fast sieben Jahre. Was Effi befürchtet und was sie schließlich fast vergessen hatte, traf ein. Durch einen Zufall findet Innstetten ein Päckchen Briefe und erkennt die Handschrift des Majors. Der Inhalt der Briefe läßt keinen Zweifel über Effis Verhältnis zu Major von Crampas. Innstetten fordert Crampas zum Duell und tötet ihn. Effi erhält die Nachricht von dem Duell und ihrer bevorstehenden Scheidung in einem Brief von ihrer Mutter.

Drei Jahre lebte Effi, von allen verstoßen, einsam in Berlin. Nur Roswitha hat sie nicht verlassen und sorgt sich mit gewohnter Liebe um ihre Herrin. Effis Sehnsucht nach ihrer Tochter gibt ihr schließlich Mut, um ein Wiedersehen zu bitten. Doch das Treffen zwischen Mutter und Tochter zeigt die ganze Grausamkeit ihres Schicksals. Innstetten hat Annie "abgerichtet." Diese Grausamkeit war mehr Strafe als Effi für ihr Vergehen verdiente. Sie bricht zusammen. Effis Gesundheitszustand verschlechtert sich. Der Arzt fürchtet, daß sie in Berlin nicht lange am Leben bleiben würde und schreibt nach Hohen-Cremmen. Vater Briest telegraphiert: "Effi, komm." Er ruft sie mit den gleichen Worten, die am Anfang des Romans als Warnung erklangen, in die Heimat zurück .

Effi lebt wieder auf. Das Glück, das sie immer in Hohen-Cremmen empfunden hat, bleibt auch diesmal nicht aus. Doch ihre Gesundheit 
verschlechtert sich und mit dem Sommer neigt sich auch ihr Leben. Sie stirbt "mit Goti und Menschen versöhnt, auch versöhnt mit ihm." (R.E., Bd. VII, S. 308).

EFFI UND INNSTETTEN :

STUDIE ZUR VERSCHIEDENHEIT DER CHARAKTERE

Die Heldin des Romans ist ein 17-jähriges Mädchen. "In allem, was sie tat," sagt der Erzähler von ihr, "paarte sich Übermut und Grazie, während ihre lachenden braunen Augen eine große natürliche Klugheit und Herzensgüte verriet." (R.E., Bd. VII, S. 58).

Mit diesen. Worten macht Fontane den Leser mit Effi bekannt. Ihre weitere Entwicklung verfolgt dann der Leser selbst. Der Schlüssel zum Charakter der Personen liegt hauptsächlich im gesprochenen Wort, in den Dialogen und Monologen des Romans.

In den Gesprächen mit Effis Freundinnen und mit ihrer Mutter, drückt sich kindliche Unbefangenheit aus und Naivität in Fragen der Liebe und Ehe. So meint sie, auf die Frage der Mutter, ob sie Innstetten nicht liebe: "Warum soll ich ihn nicht lieben? Ich liebe Hulda, und ich liebe Berta,... Ich liebe alle, die's gut mit mir meinen und gütig gegen mich sind und mich verwöhnen." (R.E., Bd. VII, S. 85). Sie ist nicht für "Musterehen." Selbst eine the ohne Liebe kann sie sich vorstellen. "Glanz und Ehre" und "Zerstreuung" aber dürfen nicht fehlen, sagt sie: "Ja, Zerstreuung, immer was Neues, immer was, daß ich lachen oder weinen muß. Was ich nicht aushalten kann, ist Langeweile." (R.E., Bd. VII, S.33).

In den Gesprächen der Eltern drückt Briest Bedenken aus, ob Effi, das "Naturkind," sich in die Rolle der Frau Landrätin wird einleben 
können. Frau Briest weiø es nicht. Sie findet Effi "mitteilsam und verschlossen zugleich." Auf die Frage ihres Mannes, ob Effi ihr nicht ihr Herz ausgeschüttet habe, antwortet sie: "Solche Generalbeichte, so alles von der Seele herunter, das liegt nicht in ihr." (R.E., Bd. VII, S. 40). Effi äußert sich auch wirklich nicht direkt, wie es um sie steht. Und doch bricht es "ungewollt und wie von ungefähr aus ihrer Seele." (R.E., Bd. VII, S. 40). Vor der Hochzeit, als sie sich weinend vor der Mutter auf die Knie wirft, im Gesprüch mit Gieshübler über Crampas, und vor allem in dem Augenblick, da Innstetten ihr von seiner Versetzung nach Berlin erzühlt:

Effi sagte kein Wort, und nur ihre Augen wurden immer größer: um ihre MundwinkeI war ein nervöses Zucken, und ihr ganzer zarter Körper zitterte. Mit einem Male aber glitt sie von ihrem Sitze vor Innstetten nieder, umklammerte seine Knie und sagte in einem Tone, wie wenn sie betete: "Gott sei Dank!" (R.E., Bd. VII, S. 191).

SchuId, Angst und Erlösung lagen in diesen drei Worten.

Obgleich Effi schon. während der Hochzeitsreise Heimweh nach Hohen-Cremmen hat, versucht sie dennoch, sich in Kessin einzuleben und ihrem Mann eine gute Frau zu sein. Trotz ihrer Angst vor dem "verwunschenen Haus" entschließt sie sich, dort wohnen zu bleiben. Sie will Innstetten die "Lächerlichkeit" ersparen, die ein Umzug bedeuten könnte. Sie klagt ihn aber an, daß er so wenig Verständnis für ihre Angst vor dem Chinesenspuk zeigt und sagt:

Ich habe dir nachgegeben und mich willig gezeigt, aber ich finde doch, daß du deinerseits teilnahmsvoller sein könntest. Wenn du wïstest, wie mir gerade danach verlangt. Ich habe sehr gelitten, wirklich sehr, und als ich dich sah, da dachte $i c h$, nun würde ich frei werden von meiner Angst. Aber du sagst mir blob, daß du nicht Lust hättest, dich lächerlich zu machen. (R.E., Bd. VII, S. 84).

Später erkennt Effi, daß Innstetten nicht nur nicht gewillt war, 
sie aus ihrer Angst zu befreien, sondern daß er den Spuk als eine "Art Angstapparat aus Kalkül" (R.E., Bd, VII, S. 140) benutzte, um sie in "Ordnung" zu halten. Sie ist empört und fühlt sich hintergangen.

Diese Unehrlichkeit verletzt sie zu tiefst.

So wenig wie sie Innstettens Unehriichkeit ertragen kann, so sehr leidet sie auch unter ihrer eigenen. Nicht die Schuld ihres Ehebruchs lastet auf ihr. Sie schäme sich nicht ihres Vergehens, sagt sie.

Ich schäme mich bloz von wegen dem ewigen Lug und Trug: immer war es mein Stolz, daw ich nicht lügen könne und auch nicht zu lügen brauche, lügen ist so gemein, und nun habe ich doch immer lügen müssen, vor ihm und vor aller. Welt. (R.E., Bd. VII, S. 230).

Als Innstetten ihr Geheimnis schlie:3lich entdeckt, endet das Lügenspiel, und sie empfindet es als Befreiung.

Die Iüge, die Effi sieben Jahre leben mußte, führt aber nie zur Selbsttäuschung. Noch im Augenblick: ihrer größten Verzweiflung, beschönigt sie nichts und bekennt sich zu ihrer Schuld. Doch die Strafe, die Innstetten ihr zukommen läbt, übertrifft das Maß ihrer Schuld und treibt sie zur Verzweiflung.

Was zuviel ist, ist zu viel. Ein Streber war er, weiter nichts. Ehre, Ehre, Ehre... und dann hat er den armen Kerl totgeschossen, den ich nicht einmal liebte. Dummheit war alles, und nun Blut und Mord. Und ich schuld...mich ekelt, was ich getan; aber was mich noch mehr ekelt, das ist eure Tugend. (R.E., Ba. VII, S. 289).

Effis Schuld war "Dummheit," menschliche Schwäche. Die Schuld der Gesellschaft aber war Grausamkeit. Obwohl Effi die grausame "Tugend" der Gesellschaft angreift, versöhnt sie sich doch mit ihren Forderungen. Sie erkennt, das Innstetten nicht anders handeln konnte.

In der Geschichte mit dem armen Crampas, (sagt sie), ja, was sollt er am Ende anders tun? Und dann, womit er mich am tiefsten verletzte, das er mein eigen Kind in einer Art $\mathrm{Ab}-$ wehr gegen mich erzogen hat, so hart es mir ankommt und so 
weh es mir tut, er hat auch darin recht gehabt. Lais ihn das wissen, dab ich in dieser Überzeugung gestorben bin. Es wird ihn trösten, aufrichten, vielleicht versöhnen. Denn er hatte viel Gutes in seiner Natur und war so edel, wie jemand sein kann, der ohne rechte Liebe ist. (R.E., Bd. VII, S. 309).

In dieser Sterbeszene leidet Effi nicht mehr an der Vergangenheit. Alles scheint weit zurückzuliegen, Kessin, der Spuk, Crampas, aber auch Berlin und ihre Auflehnung gegen Innstetten. Wehmut spricht aus ihren Worten, aber auch Versöhnung mit.ihrem Schicksal. Ihre Herzensgüte konnte selbst das harte Leben nicht in Bitterkeit umwandeln. Effi triumphiert noch in ihrem Tod über das Leben und beweist, dấ wahre Menschlichkeit sich selbst gegen das hïrteste Schicksal behaupten kann. Fontanes Zeitgenossen stellten sich fast ausschließlich auf Effis Seite. Diese Tatsache überraschte den Dichter und veranlaste ihn zu folgender Betrachtung:

Ja, Effi! Alle Leute sympathisieren mit ihr, und einige gehen, so weit, im Gegensatz dazu, den Mann als einen "alten Ekel" zu bezeichnen. Das amüsiert mich natürlich, gibt mir aber zu denken, weil es wieder beweist, wie wenig den Menschen an der sogenannten "Moral" liegt und wie die liebenswürdigen Naturen dem Menschenherzen sympathischer sind... Eigentlich ist er (Innstetten) doch in jedem Anbetracht ein ganz ausgezeichnetes Menschenexemplar, dem es an dem, was man lieben muB, durchaus nicht fehlt. Aber sonderbar, alle korrekten Leute werden schon blos um ihrer Korrekt- 9 heit willen mit Mißtrauen, oft mit Abneigung betrachtet.

Auch der moderne Leser gibt Effi den Vorzug. Das darf nicht verwundern, denn in der heutigen Zeit liegt die Betonung eher auf dem Recht des Individuums als dem der Gesellschaft.

Doch so, wie Fontane seine Charaktere gezeichnet hat, müßten wir das gleiche Mitgefïhl für Innstetten empfinden, das wir Effi entgegenbringen. Denn seine Situation ist tragischer als ihre. Effis Konflikt entsteht aus ihrem natürlichen Fühlen und dem Gesetz der Sitte, der gesellschaftlichen Ordnung. Daw sie an diesem Konflikt zu Grunde geht, 
ist beklagenswert. Ihr Tod aber hinterläßt nicht das Gefühl, daß ihr Leben sinnlos war. Innstetten dagegen kann nur auf ein "verpfuschtes" Leben zurückblicken. In ihm war das "natürliche Gefühl" durch die Pflicht zum "Gesellschafts-Etwas" verdrängt worden. Ohne das "Natürliche" aber, das nur im Einzelnen vorhanden sein kann, nicht in einem Kollektiv, ist Innstetten eben ein Menschen-Exemplar.

Baron Innstetten ist, wie Frau Briest sagt: "Ein Mann von Charakter, von Stellung und guten Sitten." (R.E., Bd. VII, S. 18). Er ist ein "Karrieremacher," der es weit in seiner Beamtenlaufbahn bringt. Vom Landrat in Kessin wird er zum Ministerialrat nach Berlin befördert und schließlich zum Ministerialdirektor.

Die gleiche Disziplin, die Innstettens Berufsleben bestimmte, herrscht auch in seinem privaten Leben. Der Erzähler beschreibt einen typischen Abend folgendermaßen:

Es war fast zur Regel geworden, daß er sich, wenn Friedrich die Lampe brachte, aus seiner Frau Zimmer in sein eigenes zurückzog. "Ich habe da noch eine verzwickte Geschichte zu erledigen." Und damit ging er. Die Portiere blieb freilich zuräckgeschlagen; so das Effi das Blättern in dem Aktenstück oder das Kritzeln seiner Feder hören konnte, aber das war auch alles. Um neun erschien dann Innstetten wieder zum Tee, meist die Zeitung in der Hand, ....Um Zehn war Innstetten dann abgespannt und erging sich in ein paar wohlgemeinten, aber etwas müden Zärtlichkeiten, die sich Effi gefallen lieb, ohne sie recht zu erwidern. (R.E., Bd. VII, S. 108).

Diese kleine Szene zeigt, daß fir. Innstetten Ordnung und Pflichterfüllung gleichbedeutend sind mit Glück. Der Ehebruch stört die Ordnung. Das Glück wird aber erst durch Innstettens Entdeckung von Effis Untreue zerstört.

Im 27. Kapitel setzt sich Innstetten im Gespräch mit wïllersdorf mit der Frage auseinander, ob der einzelne ein Recht auf Glück habe, 
oder ob das persönliche Glück vor den Forderungen der bestehenden Gesellschaftsordnung zurücktreten müsse. Er meint, er könne Effis thebruch verzeihen. Auch sei er ohne Haß gegen Crampas. Doch die Entscheidung fällt zugunsten des "tyrannisierenden Gesellschafts-Etwas" aus, das ihm keine Wahl läst. Innstetten sagt: "Man ist nicht bloß ein einzelner Mensch, man gehört einem Ganzen an, und auf das Ganze haben wir beständig Rücksicht zu nehmen, wir sind durchaus abhängig von ihm." (R.E., Bd. VII, S. 247). Aber nicht nur die "Rücksicht auf das Ganze" zwingt ihn zum Handeln; seine eigene Selbstachtung verlangt, daß die Ordnung wieder hergestellt wird. "Die Gesellschaft verachtet uns, und zuletzt tun wir es selbst und können es nicht aushalten und jagen uns die Kugel durch den Kopf." (R.E., Bd. VII, S. 247). Innstetten gehorcht also nicht nur einem äuperen Gesetz, sondern einem inneren Zwang. Gesellschaftliche Ordnung ist ihm zur inneren Notwendigkeit geworden. Wüllersdorf fawt es in folgende Worte: "Unser Ehrenkultus ist ein Götzendienst, aber wir müssen uns ihm unterwerfen, solange der Götze gilt." (R.E., Bd. VII, S. 300). Diesem "Götzen" opfert Innstetten Crampas, seine Ehe und sein Glïck. Doch die Sinnlosigkeit dieses Opfers läßt inn an der Gültigkeit der bestehenden Gesellschaftsordnung zweifeln. Selbst die Ehrenbezeugungen können die Leere in seinem Leben nicht verdrängen. Alles was er früher für gut und wünschenswert hielt, erscheint ihm jetzt als fraglich. Er möchte "aus dieser ganzen Geschichte heraus," aus seiner Existenz innerhalb der Gesellschaftsordnung. Er möchte ein neues Leben beginnen, ein Leben, das seiner individuellen Natur entspricht. In seinem "Schulmeistertum," das er sein "Eigentlichstes" nennt, glaubt er seine individuelle Natur $z u$ finden. Doch Innstetten erkennt zugleich, da: 
es nicht geht. Er kann sich nicht von dem "Ganzen" lösen, um ein Einzelleben zu führen. Er muß Wüllersdorf zustimmen, dåे es kein Entkommen gibt, und daß nichts bleibt als Resignation.

VERGLEICH DER HAUPTFIGUREN :

CHRISTINE - INNSTETTEN, EFFI - HOLK

Vergleicht man die Charaktere in den Romanen Unwiederbringlich und Effi Briest, dann fallen gewisse Parallelen ins Auge. Das Ehepaar Christine - Holk in Unwiederbringlich erscheint in der Vertauschung Innstetten - Effi in Effi Briest wieder.

Christine und Innstetten sind ernst, konservativ, pflichtbewußt und von strenger Moral. Sie beschüftigt sich eingehend mit der rechten Erziehung ihrer Kinder, er mit der Erziehung seiner kindlichen Frau. Von beiden wird gesagt, daß sie "Basedow oder Pestalozie" gewesen wären, wenn sie nicht als Innstetten oder Arne geboren wären.

Christine und Innstetten stehen "im Dienst" eines äußeren Zwanges; für sie ist es Religion, für ihn Gesellschaftsordnung. Beide empfinden den Zwang aber nicht als eine Macht von aufen. Die lange Beamtenkarriere und seine gesellschaftliche Stellung lassen Innstetten das "Gesellschafts-Etwas" als innere Notwendigkeit erkennen. Ebenso ist für Christine, durch die strenge herrenhuter Erziehung, die Religion zur inneren Notwendigkeit geworden. Sie sind also beide vorzügliche Repräsentanten einer Ordnung, die ihnen zur zweiten Natur geworden ist. Sie nehmen diese Ordnung und sich in ihr "zu schwer." Ihre Ehepartner, die eher zum "Leichtnehmen" neigen, weichen instinktiv der ihnen fremden Schwere aus und gleiten so in ihre Verfehlung. 
Durch die Verfehlung der Ehepartner aber erkennen Innstetten und Christine ihre jeweilige Ordnung, und somit ihre "zweite Natur," als fragwürdig. Sie sehnen sich nach ihrem "Eigentlichsten," ihrer reinen Menschlichkeit, und bemühen sich um eine. ehrliche Lösung ihres inneren Konflikts. Dieses Sich-Mühen sichert ihnen zwar nicht die Liebe, aber doch die Anteilnahme des Lesers.

Holk und Effi dagegen gewinnen durch ihren Charme, ihre Lebensfreude und Herzensgüte nicht nur die Liebe ihrer Mitmenschen, sondern auch die des Lesers und des Dichters. Wie ein Seufzer entflieht dem Herzen des Erzählers kurz vor Effis Tod das "Arme Effi, du hattest zu den Himmelswundern zu lange hinaufgesehen und darüber nachgedacht, und das Ende war, daß die Nachtluft und die Nebel, die vom Teich heraufstiegen, sie wieder aufs Krankenbett warfen." (R.E., Bd. VII, S. 306). Diese gefühlsbetonte Ansprache, das "Du," durchbricht die dichterische Distanz, die Fontane gewöhnlich seinen Charakteren gegenüber einhält. Im Kontrast zu Innstetten und Christine erscheinen Effi und Holk als Menschen, die natürlicher empfinden. Nicht objektive Grundsätze, sondern Gefühle bestimmen ihren Lebensweg. Ihr natürliches Gefühl verlangt nach Wärme in der zwischenmenschlichen Beziehung. Diese Wärme finden sie aber nicht in ihrer the. Effi klagt, dab Innstetten "frostig wie ein Schneemann" (R.E., Bd. VII, S. 70) sei und Holk fragt sich, was er mit einem "Eisberg" solle.

Mangel an Wärme, das Gefühl von ihren Ehepartnern nicht verstanden zu werden und das Fehlen fester Grundsätze, führen Effi und Hoilk zum Fehltritt. Sie begehen den Ehebruch impulsiv und nicht überlegt. So erscheint der Ehebruch auch nicht als ein Vergehen gegen die Moral, sondern als ein Vergehen gegen die gesellschaftliche Ordnung. 
Die Strafe, die Holk und Effi wegen ihres Vergehens zuteil wird, steht aber in keinem Verhältnis zu ihrer Schuld. Doch sieht man ihren Ehebruch als ein Mibachten der natürlichen Gesetze, dann erscheint die Strafe angemessen. Nur ein echtes Gefühl zu einer dritten Person, nicht aber Langeweile und falschverstandene Leidenschaft, könnten den Ehebruch rechtfertigen.

Fontane zeigt in allen vier Charakteren, daß der Konflikt sich aus der unveränderbaren Natur des Menschen ergibt. Die Entfremdung des Menschen von seiner eigentlichen Natur führt zur Schuld. Die Schuld führt unausweichlich zur Katastrophe, der der Mensch unterliegt. Die Katastrophe aber vergegenwärtigt den Unterliegenden ihre Entfremdung und läßt sie ihr Eigentlichstes wiedererkennen. Das neue Bewußtsein der Charaktere aber verbürgt die Bewïhrung des Menschlichen. 


\section{KAPITEL III}

\section{THEODOR FONTANES ROMANTHEORIE}

Einer genaueren Untersuchung der benutzten künstlerischen Mittel in den beiden besprochenen Romanen soll hier eine kurze Zusammenfassung von Fontanes Romantheorie vorangestellt werden.

Ein Vierteljahrhundert vor Erscheinen seines ersten Romans befaste sich Fontane in seinem Aufsatz "Unsere Iyrische und epische Poesie seit $1848^{\prime \prime}$ mit dem Begriff des Realismus. Er erklärt, was er nicht darunter versteht: "das nackte Wiedergeben alltäglichen Lebens, am wenigsten seines Elends und seiner Schattenseiten." (SW., Bd. XXI/1, S. 12). Doch er begrüßt die Erkenntnis, "daß es zunächst des Stoffes, oder sagen wir lieber des Wirklichen, zu allem künstlerischen Schaffen bedarf" (SW., Bd. XXI/1, S. 12). Die Aufgabe des "echten Realismus" sieht Fontane in der "Läuterung" des nackten Rohstoffes:

Das Leben ist doch immer nur der Marmorstein, der den Stoff $\mathrm{zu}$ unendiichen Bildwerken in sich trägt. Sie schlummern darin, aber nur dem Auge des Geweihten sichtbar und nur durch seine Hand zu erwecken. Der Block an sich, nur herausgerissen aus einem größeren Ganzen, ist noch kein Kunstwerk. (SW., Bd. $\mathrm{XXI} / 1$, S. 12).

\section{Fontane will den Realismus also nicht als reine Imitation der}

Natur verstanden sehen. Aus diesem Grunde wendet er sich auch später gegen den Naturalismus. In seiner Auseinendersetzung mit dem Bild des Iebens, wie er es in den Romanen Zolas vorfindet, schreibt er:

...es ist durchaus niedrig in Gesamtanschauung von Leben und Kunst. So ist das Leben nicht, und wenn es so wäre, so müßte der verklärende Schönheitsschleier dafür geschaffen werden. Aber dies "erst schaffen", ist gar nicht nötig; die 
Schönheit ist da, man muß nur ein Auge dafür haben oder es wenigstens nicht absichtlich verschließen. Der echte Realismus wird auch immer schönheitsvoll sein; denn das Schöne, Gott sei Dank, gehört dem Leben gerade so gut an wie das Häbliche. Vielleicht ist es noch 1 gicht einmal erwiesen, daß das Häßliche präponderiert.

Von dieser Definition des Realismus weicht Fontane nicht. In der Darstellung des "Hä3lichen" sieht er eine Verwechslung der "Misere mit Realismus." (SW., Bd. XXI/1, S. 12). Die "Misere" ist nur ein Teil des "Wirklichen" und der Künstler habe nicht das Recht, sich der Darstellung nur eines Teiles zu widmen. "Wiederspiegelung alles wịklichen Lebens, aller wahren Kräfte und Interessen" (SW, Bd. XXI/1, S. 13) ist aie Aufgabe des Künstlers. Der realistische Dichter soll also den Stoff der wirklichen Welt entnehmen. Die "Läuterung" des Rohstoffes ergibt dann die "Wiederspiegelung des wirklichen Lebens" und mit den Mitteln der Kunst entsteht eine "neue Wirklichkeit."

In der Rezension von Gustav Freytags (1875) Ahnen stellt Fontane die Frage: "Was soll ein Roman?" und gibt folgende Definition:

Er soll uns, unter Vermeidung alles Übertriebenen und $\mathrm{HäB-}$ lichen, eine Geschichte erzählen, an die wir glauben. Er soll zu unserer Phantasie und unserem Herzen sprechen, Anregung geben, ohne aufzuregen; er soll uns eine Welt der Fiktion auf Augenblicke als eine Welt der Wirklichkeit erscheinen, soll uns weinen und lachen, hoffen und fürchten, am SchluB aber empfinden lassen, teils unter lieben und angenehmen, teils unter charaktervollen und interessanten Menschen gelebt. zu haben, deren Umgang uns schöne Stunden bereitete, uns förderte, klärte und belehrte. (SW., Bd. XXI/1, S. 239f).

Was Fontane also vom Kunstwerk fordert ist, daß es das "Herz" anspräche. Durch die Empfindung des Herzens aber bleibt der Leser nicht mehr nur Zuschauer. Er wird in das Handlungsgeschehen des Romans mit einbezogen. Im Miterleben der menschlichen Schicksale setzt sich der Leser mit seinen Empfindungen auseinander. Er wird ̈̈ber sich selbst klarer und dadurch als Mensch gefördert. 
Diese theoretischen Betrachtungen über den Begriff des Realismus und die Aufgabe des Künstlers als Schöpfer einer "Welt der Wirklichkeit," sind den dichterischen Arbeiten vorangegangen.

In seinen Romanen hat sich Fontane weitgehend an diese theoretischen Forderungen gehalten. So hat er den Rohstoff zu UnwiederbringIich und Effi Briest dem Leben entnommen. Seinem "Auge" wurde im Stoff das "Bildwerk" sichtbar (die Szene mit den Worten "Effi, komm," aus der die "ganze lange Geschichte entstanden ist") und "durch seine Hand," die vershiedensten Kunstmittel, ließ er eine neue "Welt der Wirklichkeit" erscheinen.

DIE KUNSTMITIEL IN DEN ROMANEN UNWTEDERBRINGLICH UND EFFI BRIEST: GESPRÄCH, SPRACHE, WORT, BRIEF

Zu den Kunstmitteln, deren sich Fontane in seinen Romanen bedient, gehört vor allem das Gespräch. "Meine ganze Aufmerksamkeit," sagt Fontane," ist darauf gerichtet, die Menschen so sprechen zu lassen, wie sie wirklich sprechen." 11 Im "so sprechen" spiegeln sich die Charaktere und bespiegeln durch ihre Urteile andere Figuren.

Der Dichter tritt möglichst in den Hintergrund des Romangeschehens zurück und überläût "den Figuren das Wort zur Selbstdarstellung im Gespräch." 12 Die Selbstdarstellung wird im Verlauf der Handlung durch Beobachtungen undBemerkungen anderer Gestalten ergänzt und vertieft. Aus der Vielfalt der Komentare entsteht so das Gesamtbild der Charaktere. Der Erzähler greift vermittelnd durch eine oder mehrere seiner Gestalten in den Handlungsablauf ein und sichert durch das Zuordnen der verschiedenen Perspektiven des einzelnen, die Einheit der erzählten Geschichte. ${ }^{13}$ 
Dieses Erzählverfahren kann in den hier behandelten Romanen verfolgt werden. Wie schon früher erwähnt wurde, umrei3t Fontane in einer kleinen Skizze den Charakter und die Erscheinung der zuerst auftretenden Personen. Alles weitere erfahren wir dann direkt aus den Gedanken und Worten dieser Personen.

Frau Briest und Effi werden uns beim Handarbeiten vorgefïhrt: Effi unterbricht ihre Arbeit mit gymnastischen Übungen, und die Mutter schaut ihr "verstohlen" zu, um nicht zu zeigen, "wie entzückend sie ihr eigenes Kind finde." (R.E., Bd. VIT, S. 8). Aus der Reflektion der Mutter gewinnt Effi Gestalt vor den Augen des Iesers. Das kleine Gespräch, daß sich Effis "turnerischen Drehungen" und dem Entzücken der Nutter über ihre Tochter anschließt, gestattet den ersten Blick in das Wesen der Romanheldin. "Effi, eigentlich hättest du doch wohl Kunstreiterin werden müssen. Immer am Trapez, immer Tochter der Luft." Effi bestätigt die Richtigkeit dieser Bemerkung auch gleich selber. "Von wem habe ich es? Doch nur von dir." (R.E., Bd. VII, S. 9). Fontane überläßt aber nicht nur die Selbstdarstellung den Gesprächen der handelnden Personen. Er führt in den Gesprächen der schon bekannten Figuren neue Gestalten in das Handlungsgeschehen ein. So hören wir im Geplauder Effis mit ihren Freundinnen von Innstetten, bevor er selber auftritt. Auch Crampas Erscheinen wird in einem Brief Effis an inre Eltern vorbereitet, bevor er sich selber an den Ereignissen des Romans beteiligt.

ähnlich ist das Verfahren auch in Unwiederbringlich. Nach kurzer Einführung durch den Erzähler, erfahren wir aus Christines und Holks erstem Gespräch, wie verschieden sie sich zum Neubau des Schloß̂es verhalten. In den folgenden Gesprächen wird ihre Verschiedenheit von 
anderen Personen betont. Schlieblich tritt der Konflikt, der aus ihrer Verschiedenheit entsteht, im Gespräch des Ehepaares offen zu Tage.

In diesem Gespräch sehen wir ein weiteres Merkmal des Fontaneschen Erzählverfahrens. ${ }^{14}$ Christine wilnscht, daß Holk seine Vergleiche "rücksichtsvoller" wăhle, während Holk fragt: "Was läßt dich wieder so bitter sprechen, Christine.?" (R.E., Bd. VI, S. 18). Der Dialog wird zum Gespräch über das Sprechen selbst. Der Inhalt tritt oft hinter den "Ton" der Worte zurück, so daß̉ dem "Wie" der Sprache größere Bedeutung zukommt als dem "Was" der Gesprüche. In diesem Sinne äußert sich Christine über den Brief, den Holk aus Kopenhagen erhält und der neben der Dienstberufung vor allem pikante Neuigkeiten über das Hofleben enthält. "Ich kann diesen Ton nicht recht leiden und muß dir sagen, es ist der Ton, der nach meinem Gefïhl und fast auch nach meiner Erfahrung immer einer Katastrophe vorausgeht. (R.E., Bd. VI, S. 48).

Ein ähnliches SprachbewuBtsein besitzt auch Holk. Er klagt über den "spöttisch herben Ton" (R.E., Bd. VI, S. 138) in Christines Briefen. Doch auch er hat sich im "Ton" vergriffen. Sein Schwager Arne wirft ihm vor, daß aus seinen Briefen an Christine "Nüchternheit," "Verletzungen" und "giftige Pfeile" (R.E., Ba. VI, S. 186) sprechen und Holk gibt zu, "daß mir in meiner Korrespondenz mit Christine der richtige Ton schlieblich verlorengegengen ist." (R.E., Bd. VI, S. 193f).

Wie weitgehend Fontane das Schicksal des Menschen mit dem Gebrauch der Sprache verknüpft hat, sollen folgende Beispiele belegen. In Effi Briest schreibt Innstetten (nachdem er Crampas Briefe an Effi entdeckt hatte) einen Zettel an Wüllersdorf, und bittet ihn zu sich. Im Gespräch mit dem Freunde versucht er seinen Entschlu3 zum Duell und der Scheidung von Effi zu begründen. Er meint, das "Gesellschafts-Etwas" Iieße ihm 
keine Wahl. Auch die Frage der "Verjührung," die auf einen möglichen Ausweg aus seiner Situation weist, müsse er ablehnen, da sie dem gleichen gesellschaftlichen Gesetz unterstehe. Doch schlieblich bekennt Innstetten, daß nicht das "Gesellschafts-Etwas" und nicht die ungelöste Frage der "Verjährung" ausschlaggebend sind, sondern die Macht des einmal ausgedrückten Wortes. Dieses Wort läßst ihm keine andere Entscheidungsmöglichkeit. Er mus, trotz der Fragwi3rigkeit seines Vorhabens, "die Konsequenzen des einmal Gesagten" ${ }^{15}$ auf sich nehmen.

Es ist jetzt zehn Uhr. Vor sechs Stunden, diese Konzession will ich Ihnen vorweg machen, hatte ich das Spiel noch in der Hand, konnte $i c h$ noch das eine und noch das andere, da war noch ein Ausweg. Jetzt nicht mehr, jetzt stecke $i c h$ in einer Sackgasse. Wenn Sie wollen, so bin ich selber schuld daran; ich hätte mich besser beherrschen und bewachen, alles in mir verbergen, alles im eigenen Herzen auskämpfen sollen. Aber es kam zu plötzlich, zu stark, und so kann ich mir kaum einen Vorwurf machen, meine Nerven nicht geschickter in Ordnung gehalten zu haben. Ich ging zu Ihnen und schrieb Ihnen einen Zettel, und damit war das Spiel aus meiner Hand. Von dem Augenblick an hatte mein Unglück und, was schwerer wiegt, der Fleck auf meiner Ehre einen halben Mitwisser, und nach den ersten Worten, die wir hier gewechselt, hat es einen ganzen. Und weil dieser Mitwisser da ist, kann ich nicht mehr zurück. (R.E., Bd. VII, S. 248).

Die ganze Tragik des Romans wird durch die Sprache ausgelöst.

Die Zeit hat keinen Einfluß auf die Sprache. Selbst nach sieben Jahren glücklicher Ehe, trotz Effis Untreue, zerstören Crampas Briefe Innstettens Glück. Innstettens Schreiben zwingt ihn wiederum zum Handeln, und die Konsequenz ist Crampas und Effis Tod.

Aber auch in Unwiederbringlich greift die Macht des Wortes entscheidend in das Leben der Menschen ein. Holk kann einfach nicht verstehen, warum Ebba seinen Heiratsantrag ablehnt. Er war sicher, daß sie genau so fühlte wie er.

Jedes Ihrer Worte, (sagt Holk), hat sich mir in die Seele 
eingeschrieben, und Ihre Blicke sprachen es mit, und beide, Worte und Blicke, sagten es mir, daw Sie's durch alle Tage hin beklagen würden,... wenn ich Sie verließe. Ieugnen Sie's, Ebbe - das waren Ihre Worte. (R.E., Bd. VI, S. 239)

Ebba gibt ihm aber zu verstehen, das nicht ihre "Worte" an seinem Unglück schuld sind, sondern sein Unvermögen, die Worte als das zu erkennen, was sie sind, "liebenswürdige" und "törichte" Plauderei.

Auch Christine scheitert in gewisser Hinsicht an der Macht der Sprache. Sie versucht sich durch Schweigen den Problemen der the zu entziehen. So herrscht zwar Friede, doch dieser Friede trügt:

...dies ängstliche Vermeiden alles dessen, was den Frieden hätte stören können, das Abbrechen im Gespräch, wenn doch einmal ein Zufall ein heikles Thema heraufbeschworen hatte, gerade die beständige Vorsicht und Kontrolle brachte so viel Bedrückendes mit sich, das selbst die letzten Jahre vor der Katastrophe, wo das eïgentliche Glück ihrer the schon zurücklag, als vergleichsweise glückliche Zeiten daneben erscheinen konnten. (R.E., Bd. VI, S. 255f).

Welche Bedeutung Fontane der Sprache im Miteinander der Menschen zumaß, hat er durch die Prinzessin aussprechen lassen:

Die Menschen...müssen durchaus ein Unterscheidungsvermögen ausbilden, was gesagt werden darf und was nicht; wer aber dies Unterscheidungsvermögen nicht hat und immer nur schweigt, der ist nicht bloB langweilig, der ist auch gefährlich. Es liegt etwas Unmenschliches darin, denn das Menschlichste, was wir haben, ist doch die Sprache. (R.E., Bd. VI, S. 99).

Ein weiteres Kunstmittel, dabj Fontane in beiden Romanen benutzt, ist der Brief. Wir haben gesehen; daß der Brief, ähnlich wie das Gespräch, Aussagen über den Charakter der Personen macht. Im Brief wird aber auch schon Erlebtes wiedergespiegelt und durch den. Briefschreiber interpretiert. So erfahren wir von Effis und Innstettens Hochzeitsreise nur, was für unser Verständnis von Effis Leben bedeutend ist. Wichtiger aber als die reine Vermittlung von Kenntnis durch Effis Briefe, ist ihre Verwendung als Baustein in der Gesamtstruktur der 
Romane. In Unwiederbringlich sind es vor allem die Briefe, die zwischen Holkenäs und Kopenhagen verkehren, die die Einheit des Romans herstellen. ${ }^{16}$ Der erste Brief in diesem Romen ist der schon vorher erwähnte Brief von Pentz aus Kopenhagen. Aufschlußreich ist die verschiedene Reaktion der Ehepartner. Holk amüsiert sich über den Inhalt und kann nur schlecht seine Freude verbergen, daß er bald selber in Kopenhagen sein wird. Für Christine dagegen wird der Brief zum AnlaB, sich über das sittenlose Leben des Dänischen Hofes auszulassen. Durch die Reaktion auf den Brief wird der Unterschied der Charaktere weiter verdeutlicht. Gleichzeitig aber wird der Leser auch auf Holks Leben in Kopenhagen vorbereitet.

Die Briefe haben aber auch eine psychologische Funktion. Der Konflikt der theleute ist im direkten Gespräch ausgebrochen. Durch das Briefeschreiben könnte dieser Konflikt vielleicht wieder gelöst werden.

Wie bei vielen Eheleuten, so stand es auch bei den Holkschen. Wenn sie getrennt waren, waren sie sich innerlich am nächsten, denn es fielen dann nicht bloß die Meinungsverschiedenheiten und Schraubereien fort, sondern sie fanden sich auch wieder zu früherer Liebe zurück und schrieben sich zürtliche Briefe. (R.E., Bd. VI, S. 60).

Die Betonung des positiven Einflusses von Briefen auf das Verhältnis der Eheleute vor ihrer Trennung, verstärkt die Enttäuschung, die das Ausbleiben der "zärtlichen Briefe" in den Ehepartnern und im Leser entstehen läßst.

Holks erster Brief wird auch gleich ausgelassen. Wir hören nur, daß "noch ein kurzer Brief mit der Meldung seiner glücklichen Ankunft an seine Frau geschrieben werden" (R.E., Bd. VI, S. 90) muste. Die Worte "kurz" und "Meldung" zeugen eher von Pflicht, als von dem ehrlichen Bedürfnis, sich seiner Frau mitzuteilen. Der zweite Brief, der 
dann im Gegensatz lang ist und ganz in den Roman aufgenommen wird, bestätigt die Erfahrung, die der Leser schon gemacht hat. Holk berichtet ausführlich alles Unwichtige und für sein Verhältnis zu Christine völlig Belanglose. Selbst als er auf die Kinder und die Menschen in Holken äs zu sprechen kommt, bleibt er unpersönlich und kühl.

Die Form des Briefes, sein Inhalt und Ton, weist deutlich auf die fortschreitende Entfremdung der Eheleute hin. Fontane zeigt auch immer nur Holk beim Briefschreiben und Empfangen. Dieser Kunstgriff verstärkt das Gefühl der Entfremdung und eine Aussöhnung der Gestalten erscheint immer unwahrscheinlicher.

Der Brief ist aber auch ein rein äußeres Zeichen der Trennung, vor allem dann, wenn er nicht die Entfernung, die zwischen den Briefpartnern liegt, durch seinen Inhalt überbrücken kann. Dazu kommt, dấ das geschriebene Wort durch wiederholtes Lesen verschiedene Bedeutungen annehmen kann. So ist es möglich, daß Holks "Rïhrseligkeit" beim nochmaligen Lesen des Briefes schwindet, und da:3 dieselben Worte ein entgegengesetztes Gefühl hervorrufen können.

Ähnlich wie Fontane das Gespräch zum Thema eines Gesprächs werden Iieß, macht er auch den Brief zum Thema eines Briefes. Der schon zitierte Brief von Arne an Holk enthält zum grö3ten Teil eine Beurteilung der Holkschen Briefe: "Was sind das für Briefe, die Du nun schon seit sechs Wochen schreibst oder, fast ließ3e sich sagen, auch nicht schreibst." (R.E., Bd. VI, S. 185f).

Erkennt man die Bedeutung, die Fontane dem Brief als Baustein in der Gesamtstruktur dieses Romans zugewiesen hat, überrascht die Kritik, die H.H. Reuter übt: "Briefe sind es, in die sich die Verlegenheit des 
Dichters in einigen Romanen der achtziger Jahre am Schlus flïhtet - am bedauerlichsten - Unwiederbringlich." 17

Diese Kritik scheint unberechtigt. Fontane hat wiederholt gezeigt, wie es trotz der Briefe zu keiner Annäherung der Eheleute kommt. Zum Teil sind die Briefe schuld an ihrer größeren Entfremdung. Schließlich, wo Christine nicht nur die Beziehung zu den Mitmenschen verloren hat, sondern auch zum Leben, drückt der Erzähler ihr völliges Versagen durch die Unfühigkeit aus, auch nur einen Abschiedsbrief zu schreiben:

Wir fanden auch wirklich mehrere Briefbogen, deren Anredeworte zeigten, das sie den Willen gehabt hatte,...von Holk, von Arne und auch von mir, Abschied zu nehmen. Den tiberschriften an Arne und mich waren ein par Worte wie "Habe Dank" und "Wenn Du diese Zeilen liest" hinzugefügt, aber alles war wieder durchstrichen, und den Bogen mit der Anrede "Lieber Holk" fehlte auch das: (R.E., Bd. VI, S. 265).

Christine ist völlig verstummt. Sie hat das "Menschlichste," die Sprache verloren und ist deshalb schon tot bevor sie stirbt. Aus dieser Sicht muß das Ende des Romans als folgerichtig gesehen werden.

Auch in Effi Briest spielen Briefe eine wichtige Rolle. Am Anfang des Romans hören wir, daß Effi "nichts Schöneres kannte als beispielsweise den Empfang vieler Geburtstagsbriefe." (R.E., Bd. VII, S. 22). Um so überraschender ist ihre Haltung Innstetten's Briefen gegenüber während der Verlobungszeit. Während sie und Frau Briest über die Aussteuer sprechen, kommt ein Brief von Innstetten. Sie steckt ihn ruhig beiseite und die Mutter muß sie daran erinnern. "Richtig. Den hätte ich fast vergessen." (R.E., Bd. VII, S. 32). Aus diesen Worten spricht keine Freude und kein Interesse für das, was ihr künftiger Gatte zu sagen habe.

Die Briefe, die Effi nach der Hochzeit nach Hohen-Cremen schreibt, dienen hauptsächlich dazu, ihrer wachsenden Einsamkeit und ihrer 
Sehnsucht nach der Heimat Ausdruck zu geben. Dann hört plötzlich mit dem Auftreten Crampas, der in Effis letztem Brief vorgestellt wurde, das Schreiben auf. Erst nach dem Ehebruch, und nachdem Innstetten Effi von seiner Versetzung nach Berlin gesprochen hat, trifft wieder ein Brief ein. Es ist der schon erwähnte Brief von Frau Briest, der Effi eine schnelle Abreise von Kessin ermöglicht.

Während im ersten Teil des Romans alle außer einem Brief von Effi geschrieben wurden, findet sich nur noch ein Brief von ihr im letzten Teil. In diesem Brief bittet sie Innstetten, für sie bei den Kessinern Abschied zu nehmen. Andere Briefe, die Effi in Berlin und während ihres Kuraufenthalts in Ems schreibt, werden nur erwähnt, aber nicht wiedergegeben.

Die Briefe aber, die jetzt im Roman erscheinen, bedeuten immer eine tiefgreifende Veränderung in Effis Leben. Innstetten entdeckt die aufbewahrten Briefe von Crampas an Effi. Drei von diesen Briefen werden im Wortlaut wiedergegeben. Sie lassen keinen Zweifel über Effis Verhältnis zu Crampas. Alles, was vom Erzühler nur angedeutet war, und was der Leser geahnt, aber fast vergessen hatte, ist plötzlich Tatsache. Der nächste Brief, von Innstetten an die Briests, wird nur im Gespräch von den Dienstmädchen erwähnt. Erst durch Frau Briests Brief an Effi in Ems, und auch hier nicht aus der schriftlichen Wiedergabe, sondern durch Effis Reflektion, erfahren wir den Inhalt. Nur den Teil, der sich mit Effis Zukunft befaßt, können wir mitlesen. Das "Alleinsein," von dem Effi in ihrem letzten Brief an die Mutter vor dem Vergehen sprach, wird jetzt in diesem Brief zum AusgestoBensein: "Auch das elterliche Haus wird Dir verschlossen sein; wir können Dir keinen stillen Platz in Hohen-Cremmen anbieten, keine Zuflucht in unserem 
Hause." (R.E., Bd. VII, S, 267f), Die Scheidung trifft Effi schwer, der Verlust ihres Kindes hart. Doch der Verlust des Elternhauses war mehr Strafe als sie erwartet hatte.

Der Brief von Effis Kurgeführtin, der Geheimrätin Zwicker, hat zwar keinen Einfluß auf Effis Leben, ist aber als Kommentar des Erzählers von Bedeutung. Die Zwicker schreibt an eine Freundin, nachdem sie in der Zeitung von dem Duell gelesen hatte:

Es ist unglaublich - erst selber Zettel und Briefe schreiben und dann auch noch die des anderen aufbewahren! Wozu gibt es Öfen und Kamine? Solange wenigstens, wie dieser Duellunsinn noch existiert, darf dergleichen nicht vorkommen; einem kommenden Geschlechte kann diese Briefschreibepassion (weil dann gefahrlos geworden) vielleicht freigegeben werden, Aber so weit sind wir noch lange nicht. (R.E., Bd. VII, S. 271).

Die beiden letzten Briefe des Romans bringen wieder Veränderungen in Effis Leben mit sich. Geheimrat Rummschüttel schreibt nach HohenCremmen und bewirkt so Effis Rückkehr ins Elternhaus. Auf ihren Spaziergängen ist sie viel allein und wänscht, sie hätte Rollo "hier," um sie zu begleiten. Roswitha möchte ihr diesen Wunsch erfüllen und schreibt an Innstetten darüber:

Sie (Effi) sagt immer, wenn sie ins Luch oder über Feld geht: "Ich fürchte mich eigentlich, Roswitha, weil ich da so allein bin; aber wer soll mich begleiten? Rollo, ja, das ginge; der ist mir auch nicht gram. Das ist der Vorteil, daßs sich die Tiere nicht so drum kümmern." (R.E., $\mathrm{Bd}$. VII, S. 301).

Innstetten schickt Rollo nach Hohen-Cremmen. Doch Roswithas Worte haben in ihrer Schlichtheit Innstetten bewuist gemacht, wie sinnlos "diese ganze Geschichte" war. Roswithas unbedingte liebe zeugt von echter Menschlichkeit, einer Menschlichkeit, zu der Innstetten nicht mehr zurikckfinden kann. 
Wie schon in Unwiederbringlich sind auch in diesem Romen die Briefe ein wesentlicher Teil der Gesamtkonstruktion der Erzählung. Sie dienen nicht nur dazu, Nachrichten mitzuteilen, bestimmte Charakterzüge der Gestalten aufzuzeigen oder zu vertiefen, einzelne Situationen zu bespiegeln, sondern, wie hier im zweiten Teil von Effi Briest, sie lösen die Katastrophe aus und werden dadurch bestimmend für die Handlung des Romans.

\section{ÖRTLICHKEIT, SYMBOL, LEITMOTIV}

Wie gezeigt wurde, sind Gespräche und Briefe die bedeutendsten Kunstmittel der Fontaneschen Erzählweise. Doch da die Gestalten in einem Raum Leben, die sich in diesen Konversationen und Berichten verwirklichen, ist auch der Schauplatz für das Gesamtbild der Romane wichtig.

In dem schon zitierten Brief an Julius Rodenberg äber den "Stoff der Novelle" zu Unwiederbringlich und die Transponierung der "Geschichte nach Schleswig-Holstein und Kopenhagen," berichtete Fontane, wie gründlich er sich mit der Wahl der Örtlichkeiten befaßte: "Ich ging sämtliche deutsche Höfe durch, nichts paßte mir, als ich aber Nordschleswig und Kopenhagen gefunden hatte, war ich raus!" (R.E., Bd. VI, S. 464). Er bemüht sich also ganz bewußt um eine passende Örtlichkeit für das schon feststehende Handlungsgeschehen.

Wie schon das Handlungskonzept auf einer wahren Begebenheit beruht, ist auch der Sohauplatz einer wirklichen Landschaft entnommen. Dabei geht es Fontane aber nicht um eine wahrheitsgetreue Wiedergabe der Irokalitäten. Er stellt vielmehr bekannte Ortsnamen gleichwertig neben erfundene, und schafit so die jeweils spezifische Atmosphäre, 
die der Entfaltung seiner Romangestalten gemä3 ist. ${ }^{18}$ So beginnt der Roman Unwiederbringlich mit einer gensuen geographischen Angabe, die sogleich in die Beschreibung des erfundenen Schloßes übergeht. "Eine. Meile südlich von Glücksburg, auf einer dicht an die See herantretenden Düne, lag das von der gräflich Holkschen Familie bewohnte Schlo3 Holkenm äs... Es war ein nach italienischen Mustern aufgeführter Bau." (R.E., Bd. VI, S. 7). Die folgenden Einzelheiten der Schlo3architektur werden dann, einer Bildbeschreibung ähnlich, aufgezählt:

...ein aus Säulen zusammengestelltes Oblong, hinter dem sich der Unterteil des eigentlichen Baues mit seinen Wohn- und Repräsentationsräumen versteckte, während das anscheinend stark zurücktretende ObergeschoB wenig über mannshoch über die nach allen vier Seiten hin eine Vorhalle bildende Säuleneinfassung hinauswachs. (R.E., Bd. VI, S. 7).

Eine ähnlich Bildbeschreibung findet sich im Eingangskapitel zu Effi Briest. Hier sind die Anweisungen des Dichters noch genauer. "In Front des Herrenhauses zu Hohen-Cremmen...fiel heller Sonnenschein." Zur "Park- und Gartenseite hin" war ein "rechtwinklig angebauter Seitenflügel" mit einem "weis und grün quadrierten Fliesengang." "Einige zwanzig Schritte weiter, in Richtung und Lage genau dem Seitenflügel entsprechend, lief eine...Kirchhofsmauer. Fronthaus, Seitenflügel und Kirchhofsmauer bildeten ein...Hufeisen." (R.E., Bd. VII, S. 7).

In beiden Romanen geht die gedrängte Ortsskizze bald in eine Bühnenanweisung über und stellt so den Bezug zum Menschen her. Die aufgeführten Gegenstände bilden den Hintergrund für die nun beginnenden Handlungen und Gespräche der Romanfiguren. Je ein Beispiel aus Unwiederbringlich und Effi Briest soll dieses "in Szene setzen" verdeutlichen.

Man sab unter der Fronthalle, den Blick aufs Meer, den groBen EBsaal, dessen hohe Glastïr aufstand, im Rücken,...an einem anderen Tisch, hatte die Gräfin Platz genommen im 
Gespräch mit dem Seminardirektor Schwarzkoppen. Arne schritt mit seinem Schwager Holk auf den Steinfliesen auf und $a b$. (R.E., Bd. VI, S. 13).

Ähnlich sind die Regieanweisungen auch in Effi Briest:

Auch die Front des Herrenhauses gewährte bei bewölktem Himmel einen angenehmen und zugleich allerlei Zerstreuung bietenden Aufenthalt; an Tagen aber, wo die Sonne niederbrannte, wurde die Gartenseite ganz entschieden bevorzugt, besonders von Frau und Tochter des Hauses, die denn auch heute wieder auf dem im vollen Schatten liegenden Fliesengang salien, in ihrem Rücken ein paar offene, von wildem Wein umrankte Fenster, neben sich eine vorspringende Treppe, deren vier Seitenstufen vom Garten aus in das Hochparterre des Seitenflügels hinaufführten. Beide, Mutter und Tochter, waren fleibig bei der Arbeit. (R.E., Bd. VII, S. 7f).

Sobald die Personen ihren angewiesenen Platz eingenommen haben, dominiert immer die Aktion und das Gespräch.

Unwelt- und Raumgestaltung sind aber nicht allein auf diese Kulissenfunktion beschränkt. Vielmehr dienen sie zur Untermalung innermenschlicher und zwischenmenschlicher Vorgänge.

Die romantische Lage von Schloß3 Holkenäs in der "vom Weltverkehr abgelegenen Gegend" bietet den passenden Hintergrund für die friedvollen und glücklichen Jahre der Holkschen Ehe. Eine ähnliche Beziehung besteht $\mathrm{zwischen}$ der landschaftlichen Stille von Hohen-Cremmen und Effis Gefühl der Geborgenheit.

Kopenhagen dagegen mit seiner weltoffenen Atmosphäre und der leichtsinnigen Lebensführung des dänischen Hofes, sowie Kessin mit dem unheimlichen Spukhaus, reflektieren die seelische Veränderung der Hauptfiguren, ihre Verirrung und schließlich ihren Fehltritt.

Einige Landschaftsbilder haben bei Fontane auch Symbolgehalt. Die Winterlandschaft, die mit Schnee und Eis zu Schlittenfahrten und Schlittschuhlaufen einläd, signalisiert in beiden Romanen Gefahr. Die Sinnbilder des düner werdenden Eises in Unwiederbringlich und die 
dunklen Kronen des dichten Waldes, durch den Effis Schlittenfahrt führt, werden von den Romanfiguren als Zeichen der Gefahr erkannt. Diese symbolischen Bilder dienen also nicht nur der Verständigung des Lesers, sondern verhelfen den Romangestalten zur Klärung ihrer eigenen Situation. Ähnlich ist es mit dem Sinngehalt des Schloßbrandes in Unwiederbringlich und dem Schloon in Effi Briest. In einem einzigen Bild, in den zerstörenden Flammen des SchloBbrandes, spiegelt sich Holks Lebenssituation. Ebenso ist der Schloon, der im Winter zu einem Sog wird, Versinnbildlichung von Effis Schicksal. ${ }^{19}$

Einige Elemente der Raumgestaltung treten in den Romanen leitmotivisch auf. In Effi Briest finden wir schon im Eingangskapitel die Schaukel, die an mehreren bedeutenden Stellen des Romans wiedererscheint. 20 Kurz vor ihrer Hochzeit gesteht sie der Mutter, das sie sich nichts aus Schmuck mache. "Ich klettre lieber, und ich schaukle mich lieber; und am liebsten immer in der Furcht, daß es irgendwo reisen oder brechen und ich niederstürzen könnte. Den Kopf wird es ja nicht gleich kosten." (R.E., Bd. VII, S. 35). Effis Vorliebe für das Schaukeln zeugt von ihrer Ungebundenheit, ihrem Wunsch nach Freiheit und Schwerelosigkeit. Sie liebt aber auch die Gefahr, die sie immer wieder mit dem Schaukeln verbindet. Nach der Geburt, ihrer Tochter, während ihres ersten Besuches in Hohen-Cremmen, heiat es "Am liebsten aber hätte sie wie frïher auf dem durch die Luft fliegenden Schaukelbrett gestanden und, in dem Gefühle: 'Jetzt stürz ich," etwas eigentümlich Prickelndes, einen Schauer süßer Gefahr empfunden." (R.E., Bd. VII, S. 124). Auch wieder bei ihrem letzten Aufenthalt in Hohen-Cremen, nach ihrem Ehebruch, der Scheidung und ihrem Exil in Berlin, zeigt Fontane Effi noch einmal auf der Schaukel. 
Sie sprang hinauf, mit einer Behendigkeit wie in ihren jüngsten Mädchentagen, und ehe sich noch der Alte, der ihr zusah, von seinem halben Schreck erholen konnte, huckte sie schon zwischen den zwei Stricken nieder und setzte das Schaukelbrett durch ein geschicktes Auf- und Niederschnellen inres Körpers in Bewegung. Ein paar Sekunden noch, und sie flog durch die luft, und blo.3 mit einer Hand sich haltend, rib sie mit der anderen ein kleines Seidentuch von Brust und Hals und schwenkte es wie in Glück und Übermut. (R.E., Bd. VII, S. 295).

Durch dieses Erzählmittel, der leitmotivisch wiederkehrenden Schaukel, verbildlicht Fontane Effis Charakter, ihre Kindlichkeit, ihre Neigung zum Ungewöhnlichen und Gefährlichen, aber auch ihr Sich-gleich bleiben.

So wie das Schaukelmotiv Effis eigentlichen Lebensraum verbildlicht, so ist das Chinesenmotiv als Entfremdung von ihrem natürlichen Bereich zu verstehen. 21 Solange Effi mit Innstetten verheiratet ist, spukt der Chinese nicht nur in Kessin, sondern auch während der glücklichen Jahre in Berlin. Er scheint ein Symbol der unglücklichen Verbindung zweier im Grunde verschiedener Menschen zu sein.

Fontane benutzt aber nicht nur bildliche Leitmotive sondern auch sprachliche. 22 Auf die Bedeutung der Aussage, "Effi, komm," wurde schon hingewiesen. Hierher gehört auch der Lieblingsausspruch des alten Briest vom "zu weiten Feld." Es erscheint immer in Gesprächen, die Effis Ehe zum Thema haben; so kurz nach ihrer Hochzeit, wo die Mutter Bedenken über Innstettens Fähigkeit, Effi glücklich zu machen, ausdrückt; später, während Effis Besuch in Hohen-Cremmen, wo der Vater sich über Innstettens Fernbleiben verwundert. Schlieîlich endet der Roman im letzten Gespräch der Eltern mit den Worten, "Ach, Luise, laß...das ist ein zu weites Feld," (R.E., Bd. VII, S. 310) und läwt damit die Schuldfrage unbeantwortet. 
In Unwiederbringlich ist das Motiv des Unzugs von entscheidender Bedeutung. Die rein äußerliche Veränderung symbolisiert gleichzeitig einen inneren Wandel. Für Christine ist der Umzug ein düsteres Zeichen für ihr persönliches Leben. Ihr Bruder kritisiert den Umzug als etwas "Ungehöriges," etwas "Modernes." Ihm erscheint der Umzug als ein Bruch mit der Tradition. Für Holk bedeutet jeder Ortswechsel auch immer ein größeres Absterben seiner Liebe und ein Schritt näher zum Ehebruch. Symbolischen Gehalt haben auch die geschichtlichen Hinweise in diesem Roman. ${ }^{23}$ Die politischen Spannungen zwischen Dänemark und Schleswig-Holstein, die im vierten Kapitel besprochen werden, untermalen die Spannungen in der Holkschen Ehe. Die Ehescheidungen des Dänischen Königs weisen auf Christines und Holks Scheidung. Das Liebesverhältnis des Königs zur Danner, die er nicht heiratet, reflektiert Holks Affüre mit Ebba. Schließlich erscheint die seelische Entfremaung des Holkschen Ehepaares symbolisch als Verfall der gesellschaftlichen und politischen Integrität. Unwiederbringlich ist nicht nur das perönliche Glück, unwiederbringlich ist auch die alte statliche Ordnung. 
SCHLUß3:

RESIGNATION ALS AUSDRUCK DER MENSCHLICHKEIT

Die Besonderheiten der Fontaneschen Erzählweise und ihre Charakterisierungskunst liegen, wie diese Untersuchung zu zeigen versucht, im wesentlichen in der Verwendung des Gesprächs und der Briefe. Diese Kunstmittel hat Fontane bewu3t gewählt, denn er sieht in ihnen die bedeutendste Ausdrucksform der menschlichen Existenz. Im gesprochenen und geschriebenen Wort spiegelt sich das Wesen des Menschen, so wie auch sein Verhältnis zum Mitmenschen und zu seiner Umwelt. Aus dem Verhältnis des Menschen zum Mitmenschen entsteht durch die Mitteilung das gesellschaftliche Miteinander. Im Prozeß 3 des Lebens entwickeln sich aus dem Miteinander die Konventionen der Gesellschaft. Diese Konventionen prägen den Menschen und regeln das Neben- und Miteinander der Einzelnen. Wo die Konventionen erstarren und Selbstzweck werden, bildet sich das, was Fontane durch Innstetten als das "tyranisierende Gesellschafts-Etwas" bezeichnet.

Fontanes Gesellschaftskritik setzt dort ein, wo das "tyranisierende Gesellschafts-Etwas" die Menschlichkeit zu ersticken droht. Innstetten und Christine sind Menschentypen, deren Menschlichkeit fast ganz von den Prinzipien der Konventionen überdeckt ist. Ihr starres Festhalten an Grundsätzen enthält etwas Unmenschliches. Holk und Effi dagegen, obgleich auch sie von den Konventionen der Gesellschaft beeinfluit sind, haben noch Natürlichkeit. Dieser Natürlichkeit entspringt inr Vergehen. 
Fontane zeigt, indem er alle vier Gestalten als schuldig erscheinen läist, daß3 weder die gesellschaftliche Ordnung, noch die menschliche Natürlichkeit außerhalb der Ordnung im Recht sind. Er zeigt auch, wie die unvermeidliche Spannung zwischen gesellschaftlicher Ordnung und menschlicher Natürlichkeit zum Konflikt führt. Der Konflikt aber, für den es keine Lösung gibt, fordert den Menschen auf, zum Leben Stellung zu nehmen. Diese Stellungnahme drückt sich bei den Romangestalten in ihrer Resignation aus.

Die Resignation aber ist vielschichtig und zeigt sich in verschiedenen Formen. ${ }^{24}$ Bei Christine, die keinen Kompromis zwischen ihren Idealen und der bedingten Wirklichkeit des Lebens schließen kann, ist die Resignation Ausdruck des Unbewaltigten, der Verzweiflung.

Innstetten dagegen ringt sich zu der Erkenntnis durch, da.j seine Ideale, die gesellschaftliche Ordnung, nicht unbedingten Anspruch haben. Diese Erkenntnis bleibt aber in Intellektualismus stecken, denn Innstetten fehlt die Kraft des Herzens, um über sich selbst hinauszufinden und so zu einer tieferen Menschlichkeit zu gelangen, Seine Resignation ist deshalb Ausdruck des Verzichts auf ein glückliches Leben.

Während Christines und Innstettens Resignation eine negative Haltung zum Leben zeigt, wird bei Holk und Effi die Resignation zu einer positiven Lebenseinstellung. Holk und Effi erkennen ihre Schuld. Das Bewußtsein der eigenen Schuld gibt ihnen die Kraft, die Schuld ihrer Ehepartner $z u$ verstehen und $z u$ verzeihen. Im Verzeihen drückt sich aber die Erkenntnis aus, daß es 'ein Leben ohne Kompromisse nicht gibt. Holk und Effi sind zu Kompromissen bereit. Sie lehnen sich am Ende 
nicht mehr gegen die verdiente Strafe auf, sondern nehmen sie willig auf sich. Diese Willigkeit, das Leben ohne Vorurteile zu akzeptieren; ist ihre Form der Resignation. In dieser Art der Resignation zeigt sich das, was der Dichter Fontane als das Humane sieht. 
FUBNOTEN

${ }^{1}$ Conrad Wandrey, Theodor Fontane (München: C.H. Beck'sche Verlagsbuchhandlung. Oskar Beck, 1919), S. 410.

${ }^{2}$ Helmuth Iürnberger, Fontane (Reinbeck bei Hamburg: Rowohlt Taschenbuch Verlag GmbH, 1968), S. 171.

3 Briefe Theodor Fontanes, Zweite Sammlung (Hrsg. Otto Pniower u. Paul Schlenther. 2 Bände. Berlin, 1910), S. 131.

4 Nürnberger, S. 176-183.

Charlotte Jolles, Theodor Fontane (Stuttgart: B. Metzlersche Verlagsbuchhandlung, 1972). Jolles Buch ist eine Zusammenstellung zur erschienenen Fontane-Literatur mit kritischen Hinweisen auf die besprochenen Themen.

5 Theodor Fontane, Romane und Erzählungen in acht Bänden (Berlin und Weimar: Aufbau Verlag, 1969), S. 488. Diese benutzte Fontane-Ausgabe erscheint im Text abgekürzt wie folgt: R.E., Bd.__, S.

6 Theodor Fontane, Sämtliche Werke (Hrsg. Edgar Gro.3 u.a., München: Nymphenburger Verlagshandlung, 1959). Diese benutzte Fontane-Ausgabe erscheint im Text abgekürzt wie folgt: SW., Bd. XXI, S.

7 Theodor Fontane 1819-1969, Stationen seines Werkes (Hrsg. Bernhard Zeller, Marbach A.N., Schiller, Nationalmuseum, München, 1969).

8 Fontanes Briefe in zwei Bänden (Ausgewählt und erl. von Gotthard Erler, Berlin und Weimar, 1968) S. 394. Diese benutzte Fontane-Ausgabe erscheint im Text abgekürzt wie folgt: F.Br., Bd. , S.

${ }^{9}$ Zeller, s. 190.

10 Theodor Fontanes Briefe an seine Familie ( 2 Bände, Berlin, 1905), S. 35 .

${ }^{11}$ Ebd., S. 22.

${ }^{12}$ Vgl. Kurt Wölfel, "Man ist nicht bloß ein einzelner Mensch," (ZfdPh Bd. 82, 1963), S. 152-171. Zitiert nach: Theodor Fontane (Hrsg. Wolfgang Preisendanz, Darmstadt: Wissenschaftliche Buchgesellschaft, 1973), S. 335 .

${ }^{13} \mathrm{Vgl}$. Hubert ohl, Bild und Wirklichkeit: Studien zur Romankunst Raabes und Fontanes (Heidelberg: Lothar Stiem Verlag, 1968), S. 160. 
${ }^{14} V_{g l}$. Ingrid Mittenzwei, Die Sprache als Thema (Berlin, zürich: Verlag Gehlen, Bad Homburg V.D.H., 1970), S, 18.

${ }^{15} \mathrm{Vgl}$. Ohl, S. 167 .

${ }^{16} \mathrm{Vgl}$. Frances M. Subiotto, "The Function of Letters in Fontane's Unwiederbringlich." (MTR 65, 1970), S. 306-318.

${ }^{17}$ Hans-Heinrich Reuter, Fontane, Bd. II, S. 560.

${ }^{18}$ Vgl. Wolfgang E. Rost, Örtlichkeit und Schauplatz in Fontanes Werken (Berlin und Leipzig: Walter de Gruyter and Company, 1931). Rost untersucht die geographischen Örtlichkeiten und weist auf die Veränderungen hin, die Fontane in seinen Romanen vorgenommen hat.

${ }^{19}$ Vgl. Vincent J. Gunther, Das Symbol im Erzählerischen Werk Fontanes (Bonn: H. Bouvier and Company, 1967). Gunther untersucht die Symbole ausführlich und weist auch auf die Sprachsymbole hin.

20 Vgl. Peter Demetz, Formen des Relalismus: Theodor Fontane, Kritische Untersuchungen (München: Carl Hauser Verlag, 1964). Demetz untersucht das Flugmotiv in. Fontanes Werk und bespricht in diesem Zusammenhang das Schaukelmotiv in Effi Briest.

$\mathrm{Vgl}$. auch Bruno Hillibrand, Mensch und Raum im Roman: Studie zu Keller, Stifter, Fontane (München: Winkler Verlag, 1971).

${ }^{21} \mathrm{Vgl}$. Mittenzweis sprachliche Untersuchung des Chinesenmotivs.

22 Eda., Mittenzweis sprachliche Untersuchung zu "das ist ein zu weites Feld." Vgl. auch Ohl, S. 163.

${ }^{23} \mathrm{~V}_{\mathrm{g}}$. Vincent J. Gunther, S. $143 \mathrm{f}$.

${ }^{24} \mathrm{Vgl}$. Karl Richter, Resignation: Eine Studie zum Werk Theodor Fontanes (Stuttgart, Berlin, Köln, Mainz: W. Kohlhammer Verlag, 1966). Richter untersucht die verschiedenen Formen der Resignation an Hand der verschiedenen Romanfiguren. 
LITERATURVERZEICHNIS

I. WERKAUSGABEN

Theodor Fontane, Sämtliche Werke. Bände I-XXIII. Hrsg. Edgar Groß u. Kurt Schreinert u.a. München: Nymphenburger Verlagshandlung, 1959.

Theodor Fontane, Romane und Erzählungen in acht Bänden. Bände I-VIII. Berlin u. Weimar: Aufbau Verlag, 1969.

Theodor Fontane, Briefe. Bände I-IV. Berlin: Propyläen Verlag, 1963.

Briefe Theodor Fontanes, Zweite Sammlung. 2 Fände. Hrsg. Otto Pniower u. Paul Schlenther. Berlin, 1910.

Theodor Fontanes Briefe an seine Familie. 2 Bände. Berlin, 1905.

Fontanes Briefe in zwei Bänden. Ausgewählt u. erl, von Gotthard Erler. Berlin u. Weimar, 1968.

\section{DARSTELLUNGEN}

Alker, Ernst. Die deutsche Literatur im 19. Jahrhundert. Stuttgart: Alfred Droner Verlag, 1969.

Bockmann, Paul. "Der Zeitroman Fontanes." Der Deutschunterricht, 11(1959), S. 59-81.

Brinkmann, Richard. Theodor Fontane: Ujber die Verbindlichkeit des Unverbindlichen. München: R. Piper and Co. Verlag, S. 84-1.15.

Demetz, Peter. Formen des Realismus: Theodor Fontane, Kritische Untersuchungen. München: Carl Hauser Verlag, 1964.

Deutsche Romantheorien: Beiträge zu einer historischen Poetik des Romans in Deutschland. Hrsg. u. eingeleitet von Reinhold Grimm. Frankfurt am Main, Bonn: Athenäum Verlag, 1968.

Gilbert, Mary E. "Fontanes 'Effi Briest." Der Deutschunterricht, 11(1959), : S. 63-75.

Gunther, Vincent J. Das Symbol im Erzählerischen Werk Fontanes. Bonn: H. Bouvier and Company Verlag, 1967. 
Hillibrand, Bruno. Mensch und Raum im Roman: Stuaien zu Keller, Stifter, Fontane. München: Winkler Verlag, 1971.

Jolles, Charlotte. Theodor Fontane. Stuttgart: B. Metzlersche Verlagsbuchhandlung, 1972.

Killy, Walther. "Abschied vom Jahrhundert, Fontane: 'Irrungen, Wirrungen'; in Wirklichkeit und Kunstcharakter." Neun Romane des 19. Jahrhunderts. München: C.H. Beck'sche Verlagsbuchhandlung, 1963, S. 193-211.

Kricker, Gottfried. Theodor Fontane: Von seiner Art und epischen Technik. Hildesheim: Verlag Dr. H.A. Gerstenberg, 1973.

Lubbe, Hermann. "Fontane und die Gesellschaft." Literatur und Gesellschaft vom neunzehnten ins zwanzigste Jahrhundert. Hrsg. Hans Joachim Schrimpf. Bonn: Bouvier u. Co. Verlag, 1963, s. 229-273.

Lukacs, Georg. "Der alte Fontane." Werke: Deutsche Literatur in zwei Jahrhunderten. Neuwied u. Berlin: Hermann Luchterhand Verlag $\mathrm{GmbH}, 1964$, S. 452-498.

McDonald, Edward R. "Charakterdarsteliung in Theodor Fontanes 'Unwiederbringlich.'" Weimarer Beiträge, 17(1971), S. 197-205.

Meyer, Herman. "Theodor Fontane 'L'Adultera' u. 'Der Stechlin.'" Das Zitat in der Erzählkunst. Zur Geschichte und Poetik des europäischen Romans. Stuttgart: J.B. Metzlersche Verlagsbuchhandlung, 1967, S. 155-185.

Minder, Robert. "Über eine Randfigur bei Fontane." Neue Rundschau, $77(1966)$, S. $402-413$.

Mittenzwei, Ingrid. Die Sprache als Thema: Untersuchungen zu Fontanes Geselischaftsromanen. Berlin, Zürich: Verlag Gehlen Bad Homburg V.D.H., 1970 .

Mommsen, Katharina. Gesellschaftskritik bei Fontane u. Thomas Mann. Heidelberg: Lothar stiehm Verlag, 1973.

Müller-Seidel, Walter. "Gesellschaft und Menschlichkeit im Roman Theodor Fontanes." Heidelberger Jahrbiicher, Bd. IV, 1960.

Nürnberger, Helmuth. Fontane. Reinbeck bei Hamburg: Rowohlt Taschenbuch Verlag GmbH, 1968.

Ohl, Hubert. Bild und Wirklichkeit: Studien zur Romankunst Raabes und Fontanes. Heidelberg: Lothar Stiehm Verlan, 1968,

Preisandanz, Wolfgang. "Die Verklärende Macht des Humors im Zeitroman Theodor Fontanes." Humor als dichterische Einbildungskraft. München: Eidos Verlag, 1936, S. 214-241. 
Reuter, Hans-Heinrich. Fontane Bd. I \& II. München: Nymphenburger Verlagsbuchhandlung, 1968.

Reuter, Hans-Heinrich. "Entwicklung und Grundzüge der Literaturkritik Theodor Fontanes." Theodor Fontane, Schriften zur Literatur. Hrsg. Hans-Heinrich Reuter. Berlin: Aufbau Verlag, 1960, S. VIXX.

Richter, Karl. Resignation: Eine Studie zum Werk theodor Fontanes. Stuttgart, Berlin, Köln, Mainz: W. Kohlharmer Verlag, 1966.

Roch, Herbert. Fontane, Berlin und das 19. Jahrhundert, BerlinSchöneberg: Gebrüder Weiss Verlag, 1962.

Rost, Wolfgang E. Örtlichkeit und Schauplatz in Fontanes Werken. Berlin und Leipzig: Welter de Gruyter and Company, 1931.

Sakrawa, Gertrud M. "Scharmanter Egoismus. Theodor Fontanes 'Unwiederbringlich." Monatshefte (Wisconsin)61 (1969), S. 15-29.

Schillemeit, Jost. Theodor Fontane, Geist und Kunst seines Alterswerks. Zürich: Atlantis Verlag, 1961.

Schweizer, Ronald. Thomas Mann und Theodor Fontane: Eine vergleichende Untersuchung zu Stil und Geist ihrer Werke, Zürich: Juris Druck und Verlag, 1971.

Strech, Heiko. Theodor Fontane: Die Synthese von Alt und Neu. Berlin: Erich Schmidt Verlag, 1970.

Subiotto, Frances M. "The Function of Letters in Fontane's 'Unwiederbringlich." MLR, 65(1970), S. 306-318.

Thanner, Josef. Die Stilistik Theodor Fontanes: Untersuchungen zur Erhellung des Begriffes "Realismus" in der Literatur. The HagueParis: Mouton and Company, 1967.

Theodor Fontane. Hrsg. Wolfgang Preisendanz. Darmstadt: Wissenschaftliche Buchgesellschaft, 1973.

Wiese, Benno von. Der deutsche Roman. Düsseldorf: A. Bagel, 1965.

Wölfel, Kurt. "'Man ist nicht Bloss ein Einzelner Mensch,' Zum Figurenentwurf in Fontanes Gesellschaftsromanen." Zeitschrift für deutsche Philologie, 82(1963), S. 152-171.

Wandrey, Conrad. Theodor Fontane, München: C.H. Beck'sche Verlagsbuchhandlung, Oskar Beck, 1919.

Zeller, Bernhard. Theodor Fontane 1819-1969. Stationen seines Werkes. Marbach a.N.: Schiller-Nationalmuseum Dt. Literaturarchiv, 1969. 